ARTICLE

DOI: $10.1038 / s 41467-018-04569-1 /$

\title{
TLR9 activation via microglial glucocorticoid receptors contributes to degeneration of midbrain dopamine neurons
}

Layal Maatouk, Anne-Claire Compagnion", Maria-Angeles Carrillo-de Sauvage ${ }^{2}$, Alexis-Pierre Bemelmans (1) ${ }^{2}$, Sabrina Leclere-Turbant ${ }^{3}$, Vincent Cirotteau ${ }^{1}$, Mira Tohme ${ }^{4}$, Allen Beke${ }^{1}$, Michaël Trichet ${ }^{5}$, Virginie Bazin ${ }^{5}$, Bobby N. Trawick ${ }^{6}$, Richard M. Ransohoff ${ }^{7}$, François Tronche ${ }^{1}$, Bénédicte Manoury ${ }^{4} \&$ Sheela Vyas ${ }^{1}$

Inflammation is a characteristic feature of Parkinson's disease (PD). We examined the role of TLR9 and its regulation by glucocorticoid receptors (GRs) in degeneration of substantia nigra dopamine neurons (DNs). TLR9 agonist, CpG-ODN, induced DN degeneration in mice lacking GR in microglia but not in controls. TLR9 deletion reduced DN loss in neurotoxin, 1-methyl-4phenyl-1,2,3,6-tetrahydropyridine (MPTP) mouse model of PD. GR regulates TLR9 activation during MPTP neurotoxicity as TLR9 antagonist suppressed increased DN loss in microglia/ macrophage GR mutant mice. GR absence in microglia enhanced TLR9 translocation to endolysosomes and facilitated its cleavage leading to pro-inflammatory gene expression. GRdependent TLR9 activation also triggered DN loss following intranigral injection of mitochondrial DNA. Finally, microglial GR sensitivity to A53T-alpha-synuclein induced DN degeneration as well as decreased microglial GR expression observed in SN of PD brain samples, all suggest that reduced microglial GR activity in SN can stimulate TLR9 activation and DN loss in PD pathology.

\footnotetext{
${ }^{1}$ Institute of Biology Paris Seine, Gene Regulation and Adaptive Behaviors Team, Department of Neuroscience Paris Seine, Sorbonne Université, CNRS UMR 8246 \& INSERM U1130, 9 Quai Saint Bernard, F-75005 Paris, France. ${ }^{2}$ CEA, DRF, Institut François Jacob, Neurodegenerative Diseases Laboratory, Molecular Imaging Research Center (MIRCen), CNRS, CEA, Université Paris-Sud, Université Paris-Saclay (UMR9199), F-92265 Fontenay-aux-Roses, France. ${ }^{3}$ IHU-AICM-Neuro-CEB, Plateforme de Ressources Biologiques (PRB), Hôpital de la Pitié-Salpétrière, 47 Boulevard de I'Hôpital, F-75013 Paris, France. ${ }^{4}$ INEM, INSERM U1151-CNRS UMR 8253, Hôpital Necker, Université Paris Descartes, Sorbonne Paris Cité, Faculté de Médecine, 149 Rue de Sèvres, F-75005 Paris, France. ${ }^{5}$ Institute of Biology Paris Seine, Electron Microscopy Facility, Sorbonne Université FR3631, 9 Quai Saint Bernard, F-75005 Paris, France. ${ }^{6}$ Center for Organic Chemistry, Mallinckrodt Pharmaceuticals, 3600N. Second Street, B81-TI, St. Louis, MO 63147, USA. ${ }^{7}$ Third Rock Ventures, Boston, MA 02116, USA. Correspondence and requests for materials should be addressed to S.V. (email: sheela.vyas@upmc.fr)
} 
$\mathrm{M}$ icroglial reactivity is an early and characteristic feature of Parkinson's disease (PD). Increasing evidence both from clinical and animal studies point to active microglial involvement, through secretion of inflammatory mediators, in the development and progression of PD pathology $^{1-3}$. Progressive degeneration of dopamine neurons (DNs) in substantia nigra pars compacta is a hallmark of PD pathology responsible for motor symptoms ${ }^{4}$. Among brain regions, $\mathrm{SN}$ has a high density of microglia ${ }^{5}$, and work with experimental PD models indicates a selective vulnerability of DNs to inflammatory attack by reactive microglia ${ }^{6}$.

Microglia are the principle resident innate immune cells of the brain expressing diverse classes of pattern recognition receptors including all TLR (Toll-like receptors) family members ${ }^{7}$. TLRs are activated by both PAMPs (pathogen-associated molecular patterns) present in microbes and DAMPs (danger-associated molecular patterns) present in molecules released by damaged cells, which leads to an intracellular signaling cascade and transcriptiondependent inflammatory gene expression ${ }^{8,9}$. NF- $\mathrm{KB}, \mathrm{AP}-1$, and IRF are major transcriptional factors involved in orchestrating the innate immune responses upon TLR ligand binding ${ }^{10}$. The functional roles of NF- $\mathrm{kB}$ and AP-1 in microglial-mediated inflammatory response and death of DNs have been demonstrated in experimental PD, while the presence of $\mathrm{p} 65$ subunit of NF- $\mathrm{KB}$ or JNK in microglia of SN was shown in PD post-mortem studies ${ }^{11,12}$. Several lines of evidence, based on animal studies, reinforce the idea that TLRs play a role in degeneration of DNs. Intranigral injection of LPS, through TLR4 activation in microglia, induces specific loss of $\mathrm{DNs}^{13}$. Similarly, intranigral injection of poly I:C, activating TLR3 in microglia, was found to lower the threshold of vulnerability of DNs for the neurotoxin $6-\mathrm{OHDA}^{14}$. Neuronally released oligomeric forms of $\alpha$-synuclein were shown to activate microglial TLR2 ${ }^{15}$, whereas we previously showed that MPTP (1-methly-4phenyl-1,2,3,6-tetrahydropyridine) intoxication in mice significantly upregulates TLR9 as well as TLR 4, 7 and the key TLR adapter protein MyD88 ${ }^{16}$.

Although microglial-mediated inflammatory response can exacerbate or trigger DN cell death, regulatory mechanisms in these cells normally limit innate immune actions. Among the factors controlling inflammatory reaction, the nuclear receptors such as GR, PPAR- $\gamma$, LXR, or Nurrl represent an important group shown to protect DNs in experimental Parkinsonism through regulation of transcriptional activities of NF- $\mathrm{kB}$ and AP1 in microglia ${ }^{16-19}$. In our previous work on the regulatory role of GR in microglia during degeneration of DNs triggered by the neurotoxin MPTP, we found increased levels of TLR3, 4, and 9 in SN of mice lacking GR in microglia/macrophages $\left(\mathrm{GR}^{\mathrm{LysMCre}}\right.$ mutant mice) when compared to controls ${ }^{16}$, suggesting a role of GR in controlling their expression. Interestingly, in the same study, a significant upregulation of TLR9 protein in the striatum of PD post-mortem brains compared to age-matched control subjects was also observed, pointing to a putative role of TLR9 in PD pathogenesis.

In this study, first we provide further evidence for the role of microglial GR in PD pathogenesis. In the absence of GR in microglia, there is increased death of DNs following intranigral injection of AAV-A53T- $\alpha$ synuclein. The number of microgliaexpressing GR is significantly reduced in the SN of post-mortem PD compared to control brain tissue suggesting that this loss of GR in microglia could contribute to dopamine neurodegenerative process. We then show the tight regulatory role of microglial GR in TLR9 activation. This has direct consequences for the loss of DNs in SN triggered either by TLR9 agonists or by MPTP. Overall, these results indicate that reduced GR activity in microglia sensitizes microglia to TLR9 activation, which is detrimental to survival of DNs in PD pathogenesis.

\section{Results}

The role of microglial GR in PD pathology. The transcriptional activation of GR is mediated by binding of glucocorticoids (GCs) to inert GR-protein complex in the cytoplasm provoking GR translocation to nucleus. To examine whether microglial GR expression is altered during dopamine neurodegeneration in $\mathrm{PD}$, we analyzed its localization in microglia in SN post-mortem brain sections from age and sex-matched control subjects and PD patients $(n=4)$. Double immunofluorescence (IF) labeling of Ibal and GR showed GR presence in the nucleus, in processes and microglia without GR (Fig. 1a). GR expression was quantified and the result, expressed as \% total GR + Iba + microglia shows $\%$ nuclear GR localization almost reversed in PD compared to controls. Thus $74 \pm 7.2 \%$ (mean \pm s.e.m.) of GR + Iba + microglia in controls display nuclear localization, which is reduced to 27.5 $\pm 6.8 \%$ in $\mathrm{PD}(p=0.02)$, with parallel increase of GR in processes, suggesting a dysfunction of GR signaling in PD.

Alpha-synuclein is intimately involved in $\mathrm{PD}$ pathological processes that lead to degeneration of DNs in SN. Recently, AAV A53T- $\alpha$-synuclein injection in $\mathrm{SN}$ was shown to trigger degeneration of $\mathrm{DNs}^{20}$. To examine whether microglial GR is involved in regulating $\mathrm{DN}$ degeneration triggered by pathological A53T- $\alpha$ synuclein, we injected AAV-PGK-A53T- $\alpha$-synuclein in intranigral region of control mice and two microglial GR mutant lines: $\mathrm{GR}^{\text {LysMCre }}$ mice in which GR gene is not only inactivated in microglia but also in peripheral myeloid cells ${ }^{16,21,22}$ and $\mathrm{GR}^{\mathrm{CX} 3 \mathrm{CR} 1 \mathrm{CreER}}$ where long-term gene inactivation occurs specifically in microglia ${ }^{23}$. The absence of GR in microglial cells in $\mathrm{GR}^{\text {LysMCre }}$ mice has been verified previously ${ }^{16,21}$. We verified the absence of GR in GR CX3CR1CreER2 after tamoxifen injections in control and mutant mice (Supplementary Fig. 1A) and find around $80 \%$ of microglia have no GR labeling compared to controls (Supplementary Fig. 1B).

Eight weeks following adenoviral A53T- $\alpha$-synuclein injection, the mice were sacrificed and the number of tyrosine hydroxylase immunoreactive (TH-IR) neurons quantified in SN. A decrease in the number of TH-IR neurons was observed in all three genotypes $\left(p=0.02,0.017\right.$, and 0.03 for control, GR $\mathrm{R}^{\text {LysMCre }}$ and $\mathrm{GR}^{\mathrm{CX} 3 \mathrm{CR} 1 \mathrm{CreER} 2}$ mutants; vehicle vs. A53T- $\alpha$-synuclein virus injection). Importantly, there was greater decrease in $\mathrm{GR}^{\mathrm{LysM} M}$ and GR ${ }^{\mathrm{CX} 3 \mathrm{CR} 1 \mathrm{CreER} 2}$ mice compared to control GR $\mathrm{GR}^{\text {lox/loxP }}$ mice $(p=0.01$ and 0.02 , respectively, for mutants compared to control mice) (Fig. 1b). A higher density of microglia indicative of increased proliferation was seen in GR LysMCre mutant mice compared to controls (Fig. 1c). Thus, GR in microglia acts to prevent degeneration of DNs induced by pathological form of $\alpha$ synuclein.

TLR9 triggered DN loss in SN is controlled by microglial GR. In light of our previous results showing high TLR9 protein levels in the post-mortem striatum of $\mathrm{PD}^{16}$, as well as regulation of inflammatory gene transcription by GR upon TLR9 pathway stimulation $^{24}$, we also examined TLR9 levels in SN of PD. Western blot results of TLR9 protein levels and quantification with respect to actin levels in post-mortem SN tissue from three PD patients and three age-matched controls showed a decrease in full-length (FL) TLR9 protein levels (Fig. 2a, b). A second band whose size corresponds to C-terminal active form of TLR9 ${ }^{25}$ was observed (Fig. 2a). Quantification revealed an increase in Cterminal fragment in SN of PD suggesting a likelihood of TLR9 activation in SN during pathogenesis of PD (Fig. 2b).

The role of microglial GR in TLR9-inflammatory-mediated degeneration of DNs was next examined in control, GR ${ }^{\text {LysMCre }}$, and $\mathrm{GR}^{\text {CX3CR1CreER2 }}$ mice. TLR9 sensing of stretches of DNA containing the cytosine-phosphate-guanosine oligodeoxynucleotide (CpG ODN) 

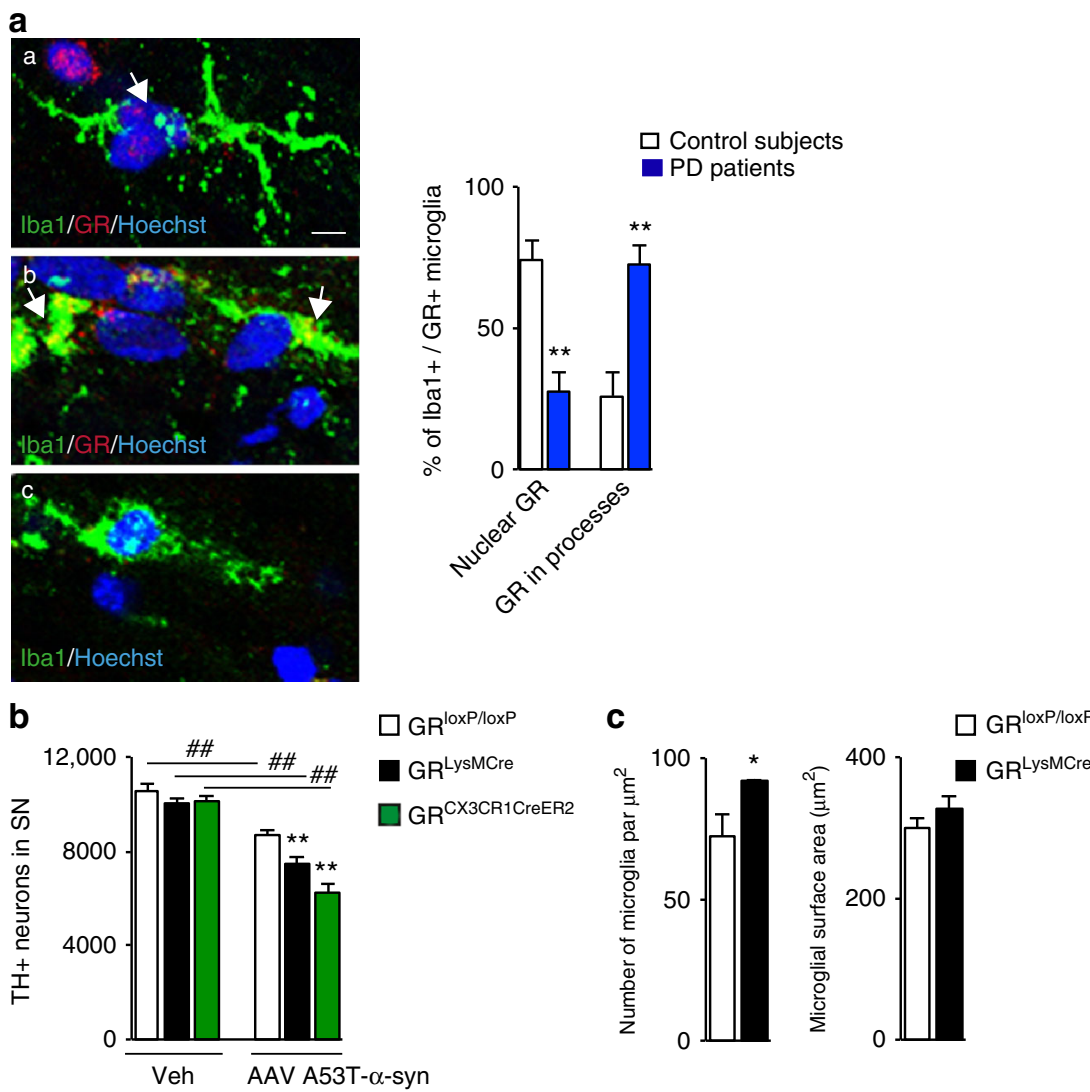

Fig. 1 Involvement of microglial GR in PD pathology. a Left: Representative confocal micrographs of Iba1 staining of microglia in SN of human post-mortem sections and GR presence: $\mathbf{a}$ in the nucleus (shown by arrows); $\mathbf{b}$ in microglia processes (shown by arrows); c absence of GR. Quantification of GR in Iba1 + microglia and GR co-localization in sections from control subjects and PD patients. Bar $=5 \mu \mathrm{m}$. The results are presented as $\%$ of GR + microglia quantified from sections of each individual; $n=4$ for control subjects and PD patients. ${ }^{\star *} p \leq 0.02$ control subjects vs. PD patients. $\mathbf{b}$ TH-IR neurons in SN of GR ${ }^{\text {loxP/ }}$ loxP,$G R^{\text {LysMCre }}$, and GR ${ }^{\text {LysMCre }}$ mice quantified 7 days after a single intranigral injection of vehicle (saline) or AAV-PGK-A53T $\alpha$-synuclein. $n=3-4$ in saline group and $n=5-7$ for AAV-PGK- $\alpha$-synuclein group, $\# \# p \leq 0.02$; vehicle saline vs. AAV-PGK-A53T $\alpha$-synuclein injection, ${ }^{\star \star} p \leq 0.02$ GR ${ }^{\text {loxP/loxP }}$ vs. $\mathrm{GR}^{\text {LysMCre }}$ or GRCX3CR1CreER2 mice after AAV-PGK-A53T $\alpha$-synuclein injection. c Quantification of microglial density and surface area in same GR ${ }^{\text {loxP/loxP }}$ and GR ${ }^{\text {LysMCre }}$ mice after AAV-PGK-A53T $\square \alpha$-synuclein injection. ${ }^{\star} p<0.05, G R^{\text {loxP/loxP }}$ vs. GR ${ }^{\text {LysMCre }}$ mice. The data presented as mean and error bars indicate s.e.m. Statistical significance determined in all by Mann-Whitney non-parametric test

motif present in bacteria is well characterized ${ }^{26}$. Control and mutant $\mathrm{GR}^{\text {LysMcre }}$ mice ( $n=5$ for each time point and genotype) were injected unilaterally with $\mathrm{CpG}$ ODN or control ODN sequence (each

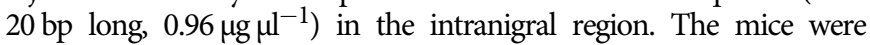
sacrificed either at 3 or 7 days after injections and total number of THIR DNs quantified. Injections of control ODN sequence in control or in mutant $\mathrm{GR}^{\text {LysMCre }}$ mice had no effect on $\mathrm{DN}$ survival when compared to saline-injected mice (Fig. 2c). In control GR ${ }^{\text {loxP/loxP }}$ mice, there was no significant decrease in the number of DNs following $\mathrm{CpG}$ ODN injection ( $p=0.48$ for 3 days and 0.09 for 7 days, CpG ODN vs. control ODN). In contrast, in $\mathrm{GR}^{\text {LysMcre }}$ mutant mice, the same dose of CpG ODN triggered significant loss of DNs at both time points of 3 and 7 days $(p<0.02$ control vs. mutant, post hoc Bonferroni/Dunn test) (Fig. 2c). To ensure that the decrease in the number of DNs is not reflecting disappearance of $\mathrm{TH}$ protein, sections were stained for Fluoro-Jade B (which labels degenerating neurons) 3 days after intranigral injection of CpG ODN. Fluoro-Jade B-positive cells were seen in the supra-nigral region, i.e., along the track of the injection (Supplementary Fig. 2A) as well as in SN (Supplementary Fig. 2B, C). Quantification of Fluoro-Jade B-positive cells in SN of control and mutant mice showed increase in degenerating neurons in GR microglial/macrophagic mutants compared to control mice $(p<0.003)$ (Fig. 2d). The parvalbumin immunoreactive neurons were quantified in $\mathrm{SN}$ region after $\mathrm{CpG}$ ODN injection both in control and
GR microglial/macrophage mutant mice to evaluate whether neurodegeneration was selective with regard to DNs. The results showed the same number of neurons in control and mutant mice (Fig. 2e). We also verified whether acute global pharmacological GR inhibition affects DN loss. Control mice were i.p. injected with vehicle or GR antagonist RU486 (30 $\left.\mathrm{mg} \mathrm{kg}^{-1}\right) 16 \mathrm{~h}$ prior to CpG ODN intranigral injection. The loss of DNs was observed $(p=0.05)$ in RU486-injected mice compared to control vehicle-pretreated mice (Fig. 2f). To verify the involvement of TLR9 in CpG ODNinduced DN death we tested TLR9 antagonist COV08-0064. The specificity and actions of COV08-0064 were reported in the peripheral tissues $^{27,28}$ and not in CNS. However, it most likely traverses the blood brain barrier as indicated by its physicochemical properties and the calcein-AM analysis in MDR1MDCKII cell line showing it is not a substrate for CNS Pgp efflux transporter (Supplementary Note 1, Supplementary Tables 1 and 2). Mutant $\mathrm{GR}^{\mathrm{LysMcre}}$ mice were i.p. injected with $80^{28}$ and 120 mg kg ${ }^{-1}$ TLR9 antagonist COV08-0064, $1 \mathrm{~h}$ prior to CpG ODN intranigral injection as well as once per day until sacrifice after 3 days. There was significant reversal of CpG ODN effect in the presence of COV08-0064 ( $p=0.03)$ indicating that COV08-0064 is antagonizing TLR9, plausibly both in macrophages and in microglia in SN as GR is inactivated in myeloid cells in GR ${ }^{\text {LysMcre }}$ mice (Fig. 2g). 
To show the involvement of GR only in microglia, CpG ODN was injected in $\mathrm{GR}^{\mathrm{CX} 3 \mathrm{CR} 1 \mathrm{CreER} 2}$ mice. In these mice, there was significant $(p=0.01)$ decrease in the number of $\mathrm{TH}+$ neurons in $\mathrm{SN}$ after CpG ODN injection compared to injection of control sequence (Fig. 2h). Thus, TLR9 activation by synthetic ligand, CpG ODN, triggers significant $\mathrm{DN}$ loss in the absence of microglial GR activity.

DNs were reported to express TLR3 and injection of its ligand poly I:C was shown to render these neurons susceptible to 6OHDA $^{29}$. Since the above results show that DN loss by $\mathrm{CpG}$ ODN is dependent on GR, we looked for any direct effect of $\mathrm{CpG}$ $\mathrm{ODN}$ on $\mathrm{DNs}$ by injecting $\mathrm{CpG} \mathrm{ODN}$ in the intranigral region of mice inactivated for GR gene specifically in DNs $\left(\mathrm{GR}^{\mathrm{DATiCre}}\right)$. Of note the absence of GR in DNs has been verified and reported previously ${ }^{30}$. Comparable numbers of DNs were found in in $\mathrm{GR}^{\text {DATiCre }}$ mutant and $\mathrm{GR}^{\text {loxP/loxP }}$ control mice following $\mathrm{CpG}$ injection (Fig. 2i) suggesting that GR in DNs does not play a role in TLR9- induced toxicity.

Previously, we showed upregulation of TLR9 during DN death triggered by MPTP ${ }^{16}$. To show that endogenous TLR9 plays a role in MPTP-triggered DN death, TLR9 gene targeted and WT mice were injected $\mathrm{x} 4$ with $20 \mathrm{mg} \mathrm{kg}^{-1} \mathrm{MPTP}$ at $2 \mathrm{~h}$ interval, a paradigm known to activate glia ${ }^{31}$. Absence of TLR9 protected DNs against MPTP $(p=0.01)$ (Fig. $2 \mathrm{j})$, suggesting that TLR9 is a

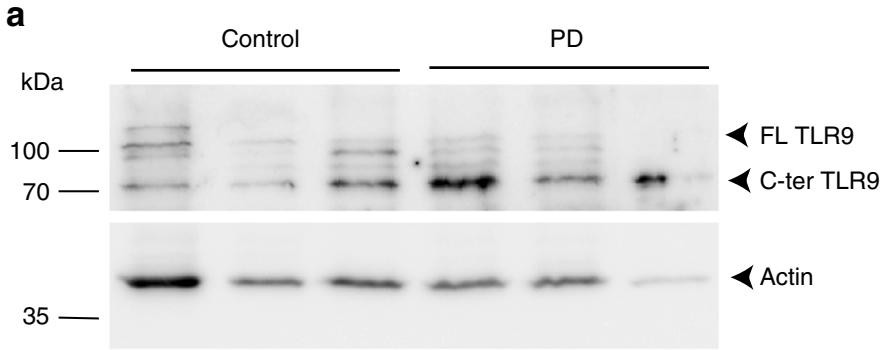

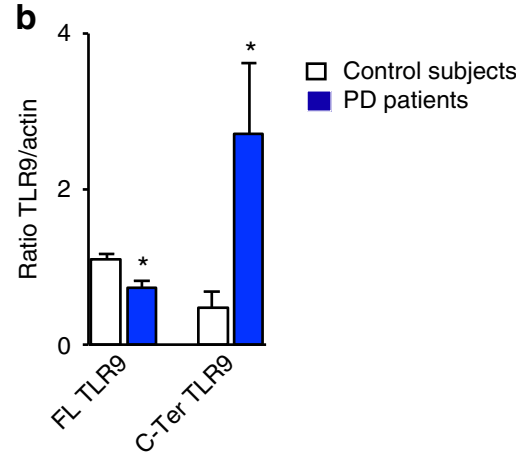

C
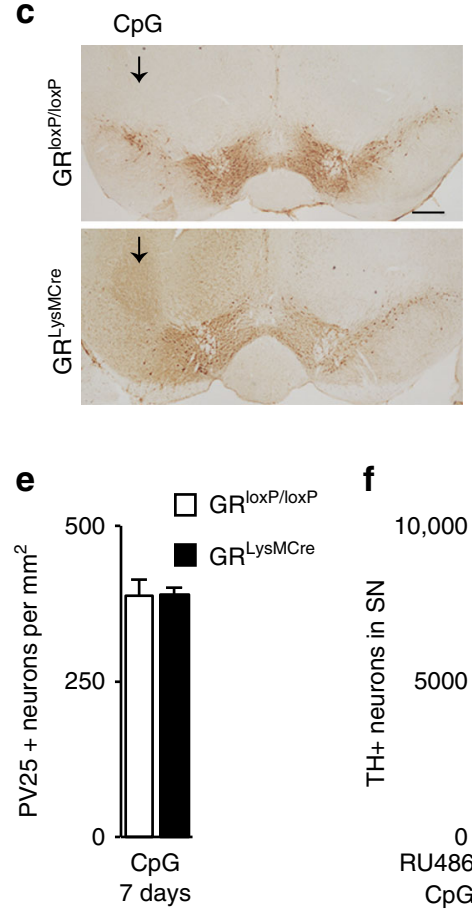

f

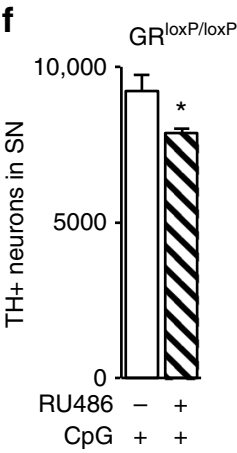

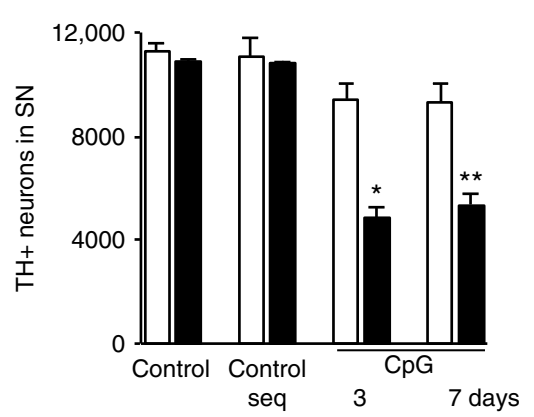

g

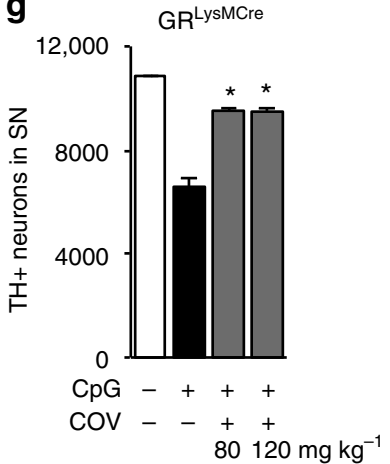

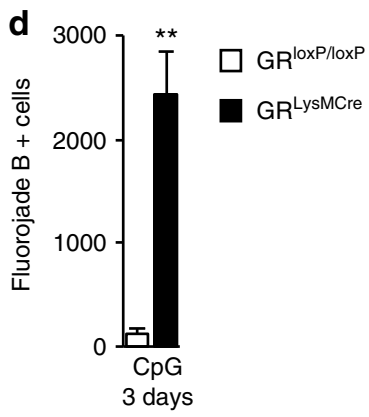

h

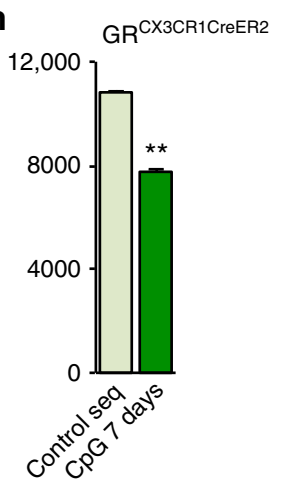

k

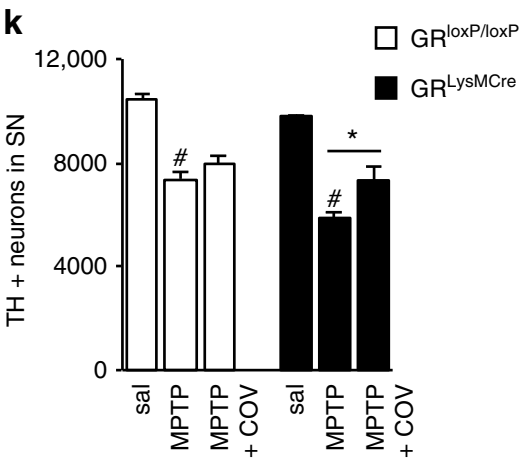


activated during dopamine neurodegeneration triggered by MPTP. To show the involvement of GR in restraining TLR9 activation during MPTP-induced dopamine neurodegeneration, control and GR ${ }^{\text {LysMcre }}$ mutant mice were injected with saline, MPTP, and with COV08-0064 prior to MPTP injection and once for 2 days consecutive following MPTP injections. The results show increased viability of DNs after COV08-006 treatment in $\mathrm{GR}^{\text {LysMcre }}$ mice compared to controls $(p=0.03)$ (Fig. $2 \mathrm{k}$ ).

Microglial GR does not affect DN loss triggered by TLR4 and TLR7. To determine whether the regulation of DN loss by microglial GR also extends to other members of TLR family, we examined the effects of TLR2, TLR4, and TLR7 activation. We performed stereotaxic injections of the TLR4 agonist, LPS $(0.5,1$,

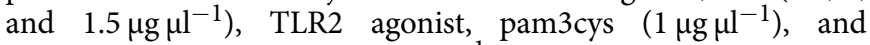
TLR7 agonist, imiquimod $\left(1 \mu \mathrm{g} \mu \mathrm{l}^{-1}\right)$ in the SN region of control $\mathrm{GR}^{\text {loxP/loxP }}$ and mutant GR $\mathrm{L}^{\mathrm{Ly} M \mathrm{Mcre}}$ mice. The mice were sacrificed 3 and/or 7 days after the injection. With LPS at $0.5 \mu \mathrm{g} \mu \mathrm{l}^{-1}$, a tendency for a decrease was observed whereas there was an inter-individual variability at $1 \mu \mathrm{g} \mathrm{l}^{-1}$ in the GR $\mathrm{G}^{\text {LysMcre }}$ mice (Supplementary Fig. 3A). As expected and previously reported $^{13}$, LPS induced significant $\mathrm{DN}$ degeneration at

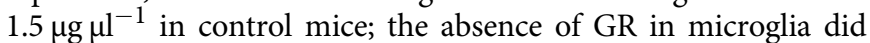
not trigger further degeneration in mutant mice $(p<0.03)$ (Supplementary Fig. 3A). The number of DNs was similar after Pam3cys injection in control and mutant mice compared to saline-injected mice, indicating that TLR2 stimulation by Pam3cys has no effect on DN survival (Supplementary Fig. 3B). On the other hand, stimulation of TLR7 by imiquimod triggered DN degeneration, which was similar in control and mutant mice ( $p=$ 0.03) (Supplementary Fig. 3C). These results suggest that among the TLR family members tested, only TLR9 activation is sensitive to microglial GR in $\mathrm{SN}$.

Deficit in motor performance following TLR9-induced DN loss. As a correlate of DN loss in SN, tests for any motor deficits were undertaken. Since our lesion was unilateral we used cylinder test as a measure of forelimb akinesia ${ }^{32}$ in control and mutant mice 12 days following CpG-ODN injection. The results showed significant reduction ( $p=0.009$ ) (approximately $35 \%$ ) in the use of contralateral paw onto surface of cylinder in mutant compared to control mice (Fig. 3a). We also used accelerating rotarod paradigm to test both for motor performance and learning in control and $\mathrm{GR}^{\text {LysMcre }}$ mutant mice just before and 1 week after intranigral $\mathrm{CpG}$ ODN injection. After habituating all mice to stay on accelerating rotarod (5-40 r.p.m.) for maximum period (3-5 $\mathrm{min})$, latency to fall on accelerating rotarod was recorded 1 week later, 3 trials/day for 3 days. The results show that over 3 days, control mice show same latency to fall, indicative of learned motor performance. However, GR ${ }^{\text {LysMcre }}$ mutant mice have impaired learned motor behavior as their latency is significantly below controls at day 1 and gradually increases attaining almost same latency as controls on day 3 (Fig. 3b). The mice were retested 1 week after intranigral CpG-ODN injection. Similar difference in latency to fall as in preinjection testing was observed between control and mutant mice at day 1 and 2 (Fig. 3c). In this regard, DN loss in $\mathrm{SN}$ is not always perceived by latency to fall in rotarod rod testing 33,34 .

GR-dependent TLR9 activation triggers pro-inflammatory state. Since TLRs trigger microglial activation, we examined microglial reactivity by Iba1 immunohistochemistry 3 days after CpG injection. Microglial surface area, as an index of glial hypertrophy or activation, was quantified both in contralateral and ipsilateral sides of $\mathrm{SN}$ of control and mutant mice following a single unilateral injection of $\mathrm{CpG}$ ODN. Microglial cells were more hypertrophied in the $\mathrm{CpG}$ ODN injected side compared to contralateral side in both controls $(p=0.04)$ and mutant $(p=$ $0.04)$ mice. There was, however, a further increase in hypertrophied microglia in microglial GR mutant mice compared to controls $(p=0.04)$ (Fig. 4a), which implies that GR expression in microglia restrains TLR9 signaling in microglia.

To examine whether exacerbated neuronal damage observed in $\mathrm{GR}^{\text {LysMCre }}$ mice was associated with inflammatory mediators, we analyzed GR transcriptional regulation of inflammatory genes. First, the results of inflammatory gene expression by RT-qPCR in SN $24 \mathrm{~h}$ after CpG ODN or control-ODN injections in control mice showed that CpG ODN has no effect on TNF- $\alpha(p=0.9)$, iNOS, MCP-1, or I KB mRNA levels. However, MyD88 mRNA levels were upregulated $(p=0.04)$ (Fig. $4 \mathrm{~b})$. In contrast, TNF- $\alpha$ $(p=0.03)$, iNOS $(p=0.03), M C P-1(p=0.02)$, and MyD88 $(p=$ $0.02) \mathrm{mRNA}$ levels were significantly increased by CpG ODN in GR $^{\text {LysMcre }}$ mutant mice (Fig. 4b), indicating that TLR9 signaling is robustly stimulated resulting in strong expression of potent inflammatory genes.

The expression of pro-inflammatory genes was verified in primary control and mutant microglial cultures treated with $\mathrm{CpG}$ ODN or CpG ODN plus GR agonist, dexamethasone (Fig. 4c). The increase in TNF- $\alpha$, iNOS, and COX2 by CpG ODN treatment

Fig. 2 Microglial GR regulates TLR9 signaling which affects DN survival in substantia nigra. a WB of TLR9 and actin in total SN homogenates from control subjects and PD patients. b Quantification of full-length (FL) and C-terminal fragment (C-ter) ${ }^{\star} p<0.05$, PD vs. control, $n=3$. Mann-Whitney nonparametric test. Full gel blots in Supplementary Fig. 4. c TH immunohistochemistry in SN of GR ${ }^{\text {LysMCre }}$ and GR ${ }^{\text {loxP/loxP }}$ mice 7 days after a single intranigral injection CpG ODN showing loss of DNs in mutants. Bar $=100 \mu \mathrm{m}$. Total number of TH-IR DNs spanning entire SN were quantified 3 and 7 days after intranigral injection of saline (sal), control sequence (c-seq), or CpG ODN. $n=5$ per group. ${ }^{\star} p<0.05,{ }^{\star \star} p \leq 0.02$, control vs. mutant, post hoc Bonferroni/ Dunn test. $\mathbf{d}$ Degenerating fluorojade-positive neurons in $\mathrm{SN}$ of controls and GR ${ }^{\text {LysMCre }}$ mice were quantified 3 days after a single intranigral injection of CpG ODN. ${ }^{\star *} p \leq 0.02$; controls vs. mutants, Mann-Whitney test, $n=5$ per group. e Quantification of parvalbumine-positive GABAergic neurons in SN of controls and GR ${ }^{\text {LysMCre }}$ mice 7 days after a single intranigral injection of CpG ODN. $\mathbf{f}$ TH-IR neurons in SN of GR ${ }^{\text {loxP/loxP }}$ mice, pretreated or not with i.p. injection of GR antagonist RU486 quantified in SN 7 days after a single intranigral injection of CpG ODN * $p<0.05$; pretreated with RU 486 vs. nonpretreated, Mann-Whitney test, $n=5$ /group. $\mathbf{g}$ TH-IR neurons in SN after injection of either 80 or $120 \mathrm{mg} \mathrm{kg}^{-1}$ COV08-0064 $1 \mathrm{~h}$ i.p. prior to stereotaxic injection of CpG ODN in GR ${ }^{\text {LysMCre }}$ mice, and $x 1$ for 3 days, mice sacrificed after 3 days. $n=4$ /group ${ }^{*} p<0.05$ pretreated with COV08-0064 vs. CpG ODN only. Mann-Whitney test. $\mathbf{h}$ Quantification of TH-IR cells in GR ${ }^{C X 3 C R 1 C r e E R 2}$ mice injected with control sequence (c-seq) or CpG ODN. ${ }^{\star *} p \leq 0.02$ control seq vs. CpG ODN, Mann-Whitney test $n=5$ group. i Quantification of TH-IR cells in SN in control and GRDATiCre mice after CpG ODN injection. $n$ $=4$ /group. $\mathbf{j}$ Saline or MPTP injections $\left(20 \mathrm{mg} \mathrm{kg}^{-1}\right)$ in wild-type (WT-C57BL/6) or TLR9 k.o. mice followed by quantification of TH-IR cells in SN. $n=3 /$ saline group and 4-5 for MPTP group. \#p<0.05 saline vs. MPTP, ${ }^{\star \star} p \leq 0.02$ WT MPTP vs. TLR9 k.o MPTP, Mann-Whitney test. k Quantification of TH-IR cells in SN 7 days after saline (sal), MPTP $\left(18 \mathrm{mg} \mathrm{kg}^{-1}\right)$, or MPTP + COV08-0064 treatment in GR ${ }^{\text {loxP/loxP }}$ and GR ${ }^{\text {LysMCre }}$ mice. $n=3 /$ saline group, $n=5 /$ MPTP group, $n=5 /$ MPTP + COV08-0064 control group, and $n=3 /$ MPTP + COV08-0064 mutant group. \#p $<0.05$ saline vs. MPTP and ${ }^{\star \star} p \leq 0.02$ MPTP vs. MPTP + COV08-0064 group, Mann-Whitney test. All data are presented as mean and error bars indicate s.e.m 


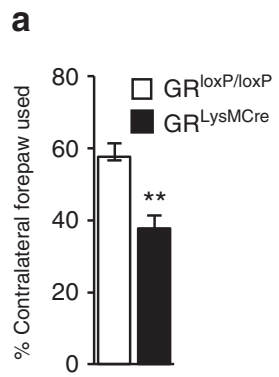

b

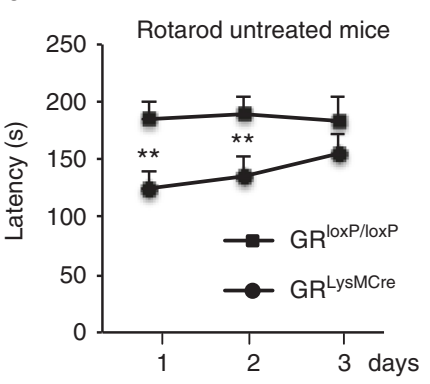

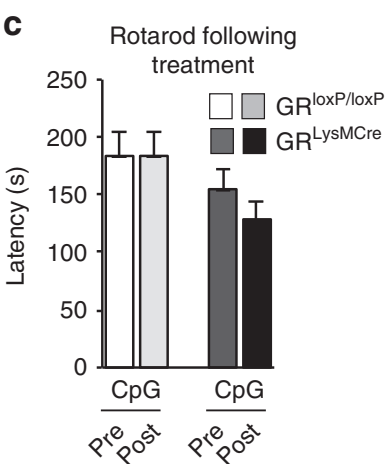

Fig. 3 Motor behavior tests in control and GR ${ }^{L y s M c r e}$ mice before and after unilateral intranigral CpG-ODN injection. a Analysis of cylinder test: \% of contralateral forelimb touch onto cylinder surface in an upright position out of 20 total ipsilateral and contralateral touches after unilateral CpG-ODN injection. ${ }^{\star \star} p<0.01$ control vs. mutant. $\mathbf{b}$ After habituation on rotarod, the control and mutant mice were scored for latency to fall on accelerating rotarod. ${ }^{\star \star} p<0.01$ control vs. mutant. c Mice retested on accelerating rotarod 1 week after CpG-ODN intranigral injection. The control mice at day 3 showed same latency as during preinjection testing whereas there was a small non-significant decrease in latency with mutant mice. $n=9$ for each group. All data are mean of three trials with error bars as s.e.m., with Mann-Whitney non-parametric test for statistical significance

was attenuated by dexamethasone in control but not mutant cultures.

TLR9 traffic to lysosomes enhanced in GR-depleted microglia. To understand how GR regulates TLR9-induced DN death, in comparison with other TLRs that also elicit inflammatory gene expression, we hypothesized that GR may have a role on upstream processes of TLR9 activation, in particular at the level of TLR9 receptor trafficking or at the level of TLR9 cleavage. TLR9 is normally retained in the endoplasmic reticulum (ER) associated with UNC93B1 $1^{35,36}$. Upon stimulation with CpG ODN, TLR9 translocates to endolysosomal compartment in UNC93B1dependent fashion, where it undergoes proteolytic cleavage by $\mathrm{pH}$-dependent proteases, notably cathepsins and asparagine endopeptidase (AEP) to yield an active C-terminal fragment, which is competent for signaling $25,37,38$.

To gain insight into the mechanisms underpinning the modulation of TLR9 signaling by GR, we used in vitro primary microglial cultures prepared from cortices of P1 control and mutant pups. The cells were transfected with TLR9-GFP expression plasmid using Amaxa nucleofector system, with transfection efficiency of $\approx 40 \%$. We analyzed co-localization of TLR9-GFP with early endosomal marker, EEA1 (Early Endosome Antigen 1) and lysosomal marker LAMP1 by confocal microscopy. Results of quantification of co-localized fluorescence signals showed that in the steady state there is little localization of TLR9-GFP with EEA1 or LAMP1-positive compartments both in control and mutant microglia confirming previous reports in dendritic and macrophage cell types (Fig. 5a). Treatment of control and mutant microglial cultures for $1 \mathrm{~h}$ with CpG ODN significantly increased TLR9-GFP localization both in early endosomes $\left(\mathrm{EEA1}^{+}\right.$, control cells $p=0.004$ and mutant cells $p=0.02$ ) and lysosomes (LAMP1 ${ }^{+}$, control cells $p=0.008$ and mutant cells $p=0.002$ ). The trafficking of TLR9-GFP in LAMP1 + compartment was significantly different between microglia from control and mutant pups following treatment with $\mathrm{CpG}$ ODN ( $p=0.001)$. Indeed, $28 \%$ of total TLR9-GFP co-localized with LAMP1 ${ }^{+}$lysosomes in mutant microglia compared to only $14 \%$ in control microglia (Fig. 5a).

We also analyzed the expression of UNC93B1 mRNA whose protein product is involved in TLR9 trafficking and found an increase in the mRNA levels in SN of GR ${ }^{\text {LysMcre }}$ mice compared to control $\mathrm{GR}^{\operatorname{loxP} / \text { loxP }}$ mice $24 \mathrm{~h}$ after intranigral $\mathrm{CpG}$ ODN injection. The expression of progranulin, whose protein product gives rise to granulins, is a co-factor for TLR9 ${ }^{39}$, was also elevated in mutant mice ( $p=0.02$ for progranulin and for UNC93B1; control vs. mutant mice) (Fig. 5b). Altogether, these results indicate the role of GR in regulating TLR9 translocation to endolysosomal compartment.

GR controls processing and AEP-dependent TLR9 activation. To examine the involvement of cathepsins and AEP in TLR9 processing in mutant vs. control microglia, we first analyzed, by RT-qPCR, the expression of cathepsins B, D, K, L, and S that can cleave TLR9 ${ }^{40}$ and found an increase only for cathepsin $S$ ( $p=$ 0.02) (Fig. 6a). Western blot analysis of protein levels of cathepsins $\mathrm{B}, \mathrm{S}$, and $\mathrm{K}$ showed no change in $\mathrm{CpG}$ ODN-injected ipsilateral SN of mutant compared to control mice (Fig. 6b). Thus, we checked for the possible involvement of AEP ${ }^{38}$ in GRmediated regulation of TLR9 activity. We pretreated control and AEP knockout mice with GR inhibitor RU486 (Fig. 1) and then injected $\mathrm{CpG}$ ODN in intranigral region. $\mathrm{TH}+$ neurons in $\mathrm{SN}$ were quantified 7 days after CpG ODN injection in control and $\mathrm{AEP}^{-1-}$ mice. The sensitivity to TLR9-induced DN loss observed after RU486 pretreatment in control mice was not observed in mice lacking AEP (Fig. 6c), indicating that cleavage of TLR9 by AEP is an important step in TLR9-induced DN toxicity.

Lysosomal changes in GR mutant microglia. Given that TLR9 cleavage is $\mathrm{pH}$ dependent, we analyzed the acidity of lysosomal compartment by treatment of control and mutant microglial cells with Lysotracker, a weakly basic amine fluorescent probe that accumulates in acidic compartments such as lysosomes. The microglial cultures incubated with Lysotracker were imaged and the mean fluorescence intensity of fluorescence-positive lysosomes, an index of the internal $\mathrm{pH}$ of the lysosomes, was quantified. The results showed increased mean fluorescence intensity in GR mutant microglial cultures compared to similarly treated control cultures $(p=0.003)$ (Fig. 7a).

Increased Lysotracker staining in GR mutant microglia may be a result of either an overall increase in the lysosomal biogenesis or more acidic lysosomes. TFEB transcription factor is a master regulator of lysosomal biogenesis. We analyzed TFEB levels in the nuclear and cytoplasmic fractions of primary microglial cultures prepared from control and mutant pups and found higher basal levels in the nuclear fraction of GR mutant cultures mirrored by a decrease in the cytoplasmic fraction (Fig. 7b). We also analyzed the expression of Ccln7, mcoln1 (TRPML1), ATP6v1h, HEXA, and lamp1 genes regulated by $\mathrm{TFEB}^{41}$ in control and GR mutant microglial cultures and found an increase in the mRNA level of 
a

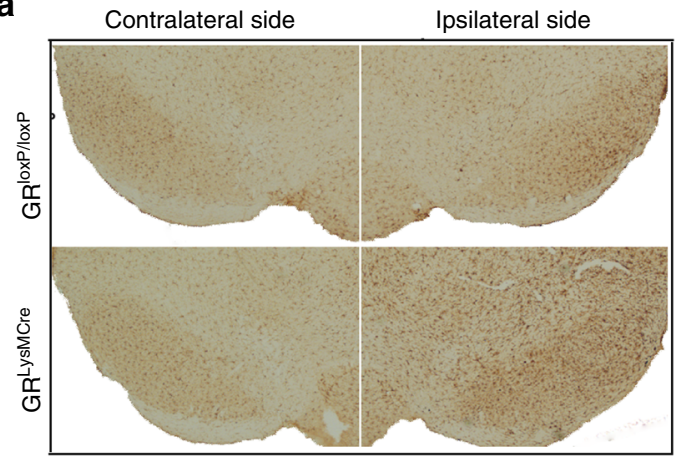

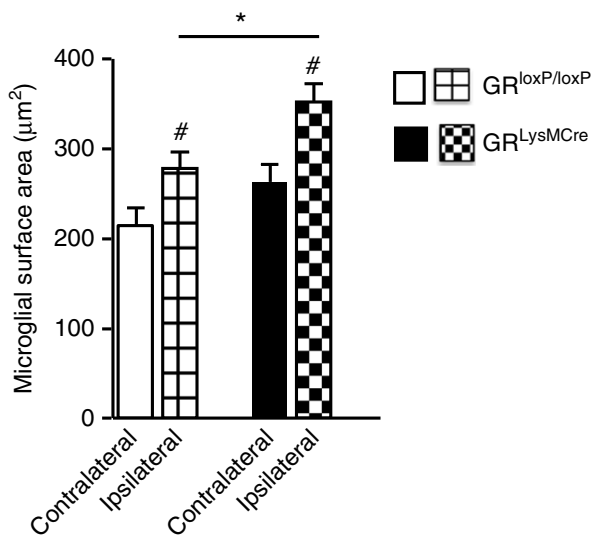
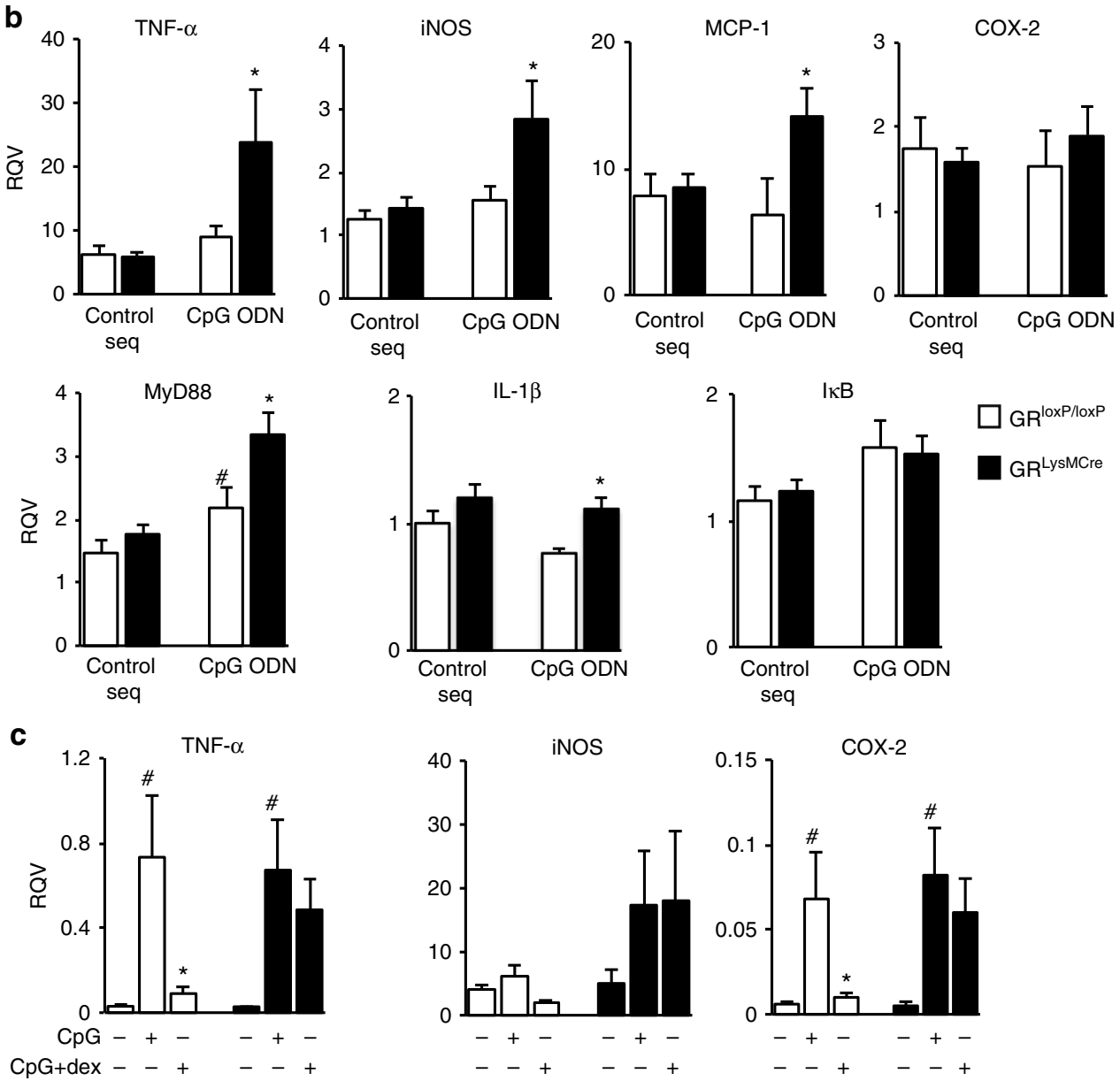

Fig. 4 Microglial GR regulates microglial reactivity and pro-inflammatory gene expression after CpG ODN injection. a Immunohistochemistry of Iba-1 7 days following CpG ODN injection both in controls and mutant mice. Quantification of microglial surface area in SN on contra-lateral and ipsi-lateral sides of $\mathrm{GR}^{\mathrm{LysMCre}}$ mutant and control mice a.u: arbitrary units. ${ }^{\star} p<0.05$ control vs. mutant; $\# p<0.05$ ipsilateral vs. contralateral side. $n=5 /$ group. $\mathbf{b}$ In vivo analysis of inflammatory gene levels by RT-qPCR $24 \mathrm{~h}$ after $\mathrm{CpG}$ ODN injection in control and GR ${ }^{\text {LysMCre }}$ mice. The RQV (relative quantitative value) was calculated using HPRT gene as internal control and compared to contralateral non-injected side. Increased levels are observed in mutants compared with controls at 24-h time point. ${ }^{*} p<0.05$, control vs. GR ${ }^{L y s M C r e}$ mutant $C p G$-injected mice, $n=4-5$. $\mathbf{c}$ In vitro analysis of pro-inflammatory gene levels by RTqPCR in control and mutant microglial cultures 40 min after control sequence (c-seq), CpG, or CpG + dexamethasone (dex) treatment. The RQV was calculated using beta-microglobulin as internal control. The data are from three independent experiments. \#p $<0.05 \mathrm{c}-\mathrm{seq}$ vs. CpG, ${ }^{\star} p<0.05 \mathrm{CpG}$ vs. CpG + dex. All data presented are mean and error bars indicate s.e.m. The statistical significance determined by Mann-Whitney non-parametric test

lamp1 in the mutant microglial cells compared to controls $(p=$ 0.05 ) (Fig. 7c). To examine whether GR absence in microglia affects lysosome numbers or size, we undertook electron microscopy analyses of lysosomes of control and mutant microglial cells (Fig. 7d). Lysosome numbers and size were quantified in 20 control and mutant cells using ImageJ. Heterogeneity in both numbers of lysosomes ranging from 8 to 120 per $100 \mu \mathrm{m}^{2}$ and individual lysosomal size ranging from 0.01 to $0.8 \mu \mathrm{m}^{2}$ was observed in both control and mutant cells. Nevertheless, as shown in Fig. 7e, mutant cells present a significant increase in the number of lysosomes (median $53 \pm 32$ lysosomes per $100 \mu^{2}$ ) compared to control cells (median $32 \pm 23$ lysosomes per $100 \mu \mathrm{m}^{2}$ ). We also analyzed the size 
a TLR9-GFP- DAPI TLR9-GFP- EEA1 TLR9-GFP- LAMP1

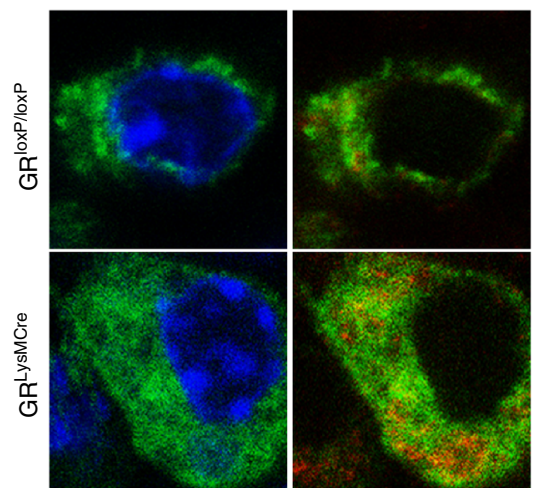

No treatment

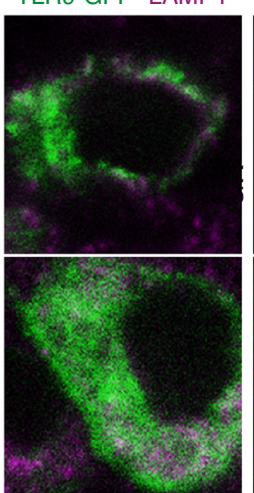

$\cos 20$

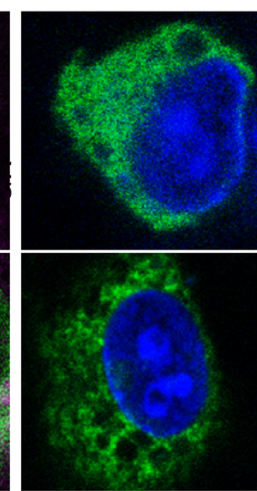

b

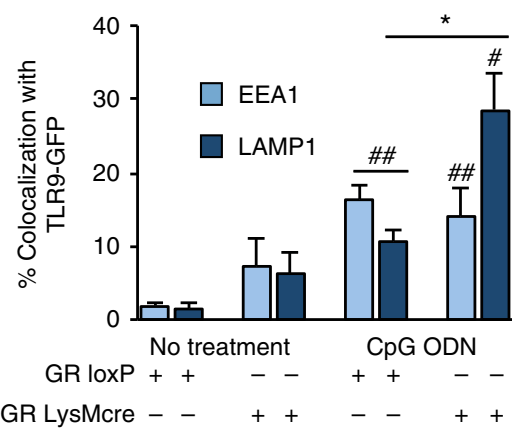

RGN

C

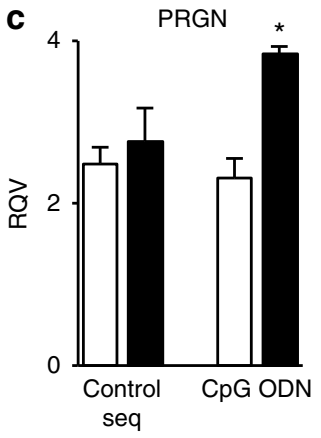

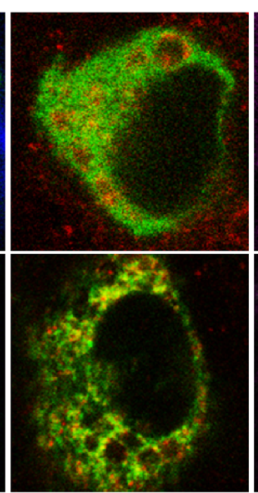

CpG ODN

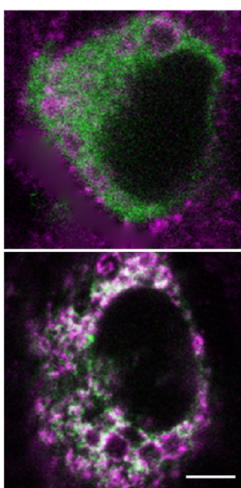

UNC93B1

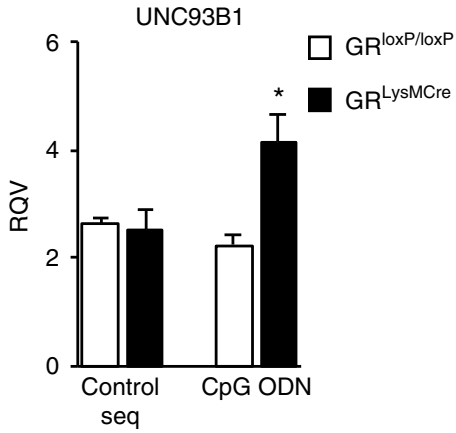

Fig. 5 TLR9 translocation to lysosomes is augmented in the absence of microglial GR. a Control (C) and mutant (M) primary microglial cells were transfected with TLR9-GFP by nucleofection and $24 \mathrm{~h}$ later stimulated or not with CpG-ODN for $1 \mathrm{~h}$. The cells were fixed and stained with specific antibodies for EEA1 (Early Endosome Antigen 1) and LAMP1 (lysosomal marker). Bar $=2 \mu \mathrm{m}$. b Quantification of TLR9-GFP/EEA1 or LAMP1 co-localization was performed using ImageJ software, from three independent experiments. The results represent the percentage of co-localization of TLR-9 GFP with either EEA1 or LAMP1. ${ }^{*} p<0.05$ Control CpG ODN vs. Mutant CpG ODN; \# non-treated vs. CpG ODN. c RT-qPCR measurement of progranulin (PRGN) and UNC93B1 mRNA levels in SN of control and mutant mice $24 \mathrm{~h}$ after control sequence or CpG ODN injection. ${ }^{*} p<0.05$ control vs. GR ${ }^{L y s M C r e}$ mice, $n=$ 4-5 per group. Data presented are mean with error bars as s.e.m., Mann-Whtiney test was used statistical significance

of lysosomes in both conditions (Fig. 7f). Comparison of the two distributions with sizes ranging from $<0.02$ to $>0.02 \mu \mathrm{m}^{2}$ revealed a significant difference in the distribution of the lysosome size $\left(\chi^{2}=84.81\right.$, df $\left.10, p<5 \times 10^{-14}\right)$. In the mutant cells, there is slight diminution of the number of lysosomes within the $0.02-0.12 \mu \mathrm{m}^{2}$ range surface, while number of lysosomes with surface $<0.02 \mu \mathrm{m}^{2}$ is increased by 2.4 -fold.

Mitochondrial DNA triggers DN loss through TLR9 activation. One of the endogenous ligands reported to activate TLR9 is mitochondrial DNA, which contains CpG DNA repeats, and is released upon cell injury ${ }^{42}$, or which escapes from autophagic degradation $^{43}$. We reasoned that mitochondrial DNA is a plausible endogenous candidate of GR-dependent TLR9 activation in the context of DN neurodegeneration, as there is substantial literature on mitochondrial and autophagic dysfunction in PD pathology ${ }^{44,45}$. Mitochondrial DNA (1 or $5 \mu \mathrm{g} \mu \mathrm{l}^{-1}$ ) was injected in the nigral region of control and GR ${ }^{\text {LysMcre }}$ mutant mice and $\mathrm{TH}+\mathrm{DNs}$ in SN were quantified after 7 days. Whereas in the control mice, mitochondrial DNA had no effect, there was DN loss in $\mathrm{GR}^{\text {LysMcre }}$ mutants (Fig. $\left.8 \mathrm{a}\right)(p=0.02$; control vs. mutant mitochondrial DNA-injected mice). To test whether mitochondrial DNA injection activates microglia, microglial reactivity was analyzed in SN of mice injected with $1 \mu \mathrm{g}$. The results showed increased hypertrophy in ipsilateral side in mutant compared to control mice $(p=0.02)$ (Fig. $8 b)$. To verify that mitochondrial DNA-mediated DN neurodegeneration following GR inhibition is elicited by TLR9, we injected $1 \mu \mathrm{g}$ mitochondrial DNA in TLR9 knockout mice pretreated or not with RU486. The results of THIR DN neuron quantification (Fig. 8c) show that there is no loss of DNs in TLR9 knockout mice pretreated with RU486 (cf. Fig. 1 control mice pretreated with RU486), indicating that DN loss by mitochondrial DNA occurs through TLR9.

\section{Discussion}

We observed a significant reduction in the number of microglia with nuclear GR localization in post-mortem SN of PD suggesting that diminution of GR activity likely promotes DN loss through stimulation of microglial inflammatory signaling cascades. This is corroborated by the results of A53T a-synuclein injection in mice inactivated for GR in myeloid cells or microglia, which resulted in increased degeneration of DNs. Inflammatory signaling cascades initiated by ligation of TLRs are well characterized, as well, microglia are known to express all the members of TLR family. Previously, we reported high TLR9 levels in striatum of postmortem PD brain tissue ${ }^{16}$. Analysis of TLR9 levels in SN of PD show increased level of C-terminal active fragment of TLR9 protein, suggesting that TLR9 processing may be augmented in SN of PD. These TLR9 protein changes in SN of PD prompted us to examine in mouse models whether TLR9 affects DN survival in $\mathrm{SN}$ and elucidate the mechanism(s) through which microglial GR acts to regulate TLR9 inflammatory cascade.

In both $\mathrm{GR}^{\text {LysMcre }}$ and GR ${ }^{\mathrm{CX} 3 \mathrm{CR} 1 \mathrm{CreER} 2} \mathrm{GR}$ mutant mice, intranigral injection TLR9 ligand, CpG ODN, resulted in 
a

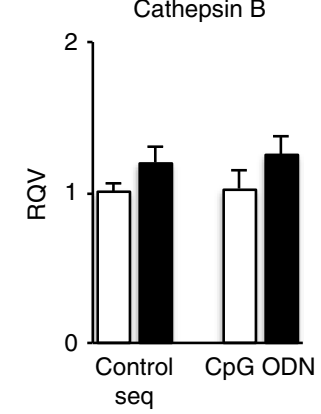

b

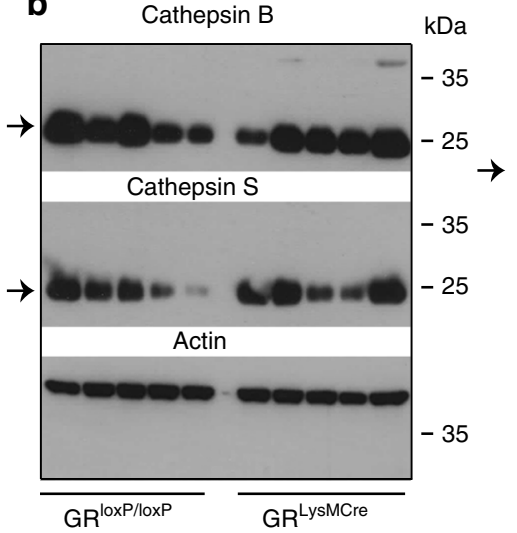

Cathepsin D

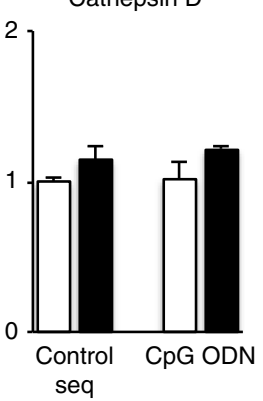

Cathepsin K

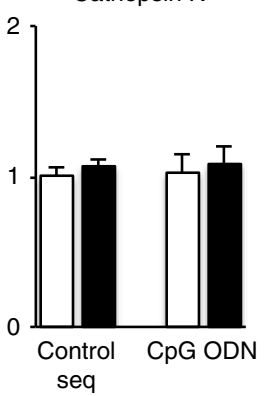

Cathepsin K

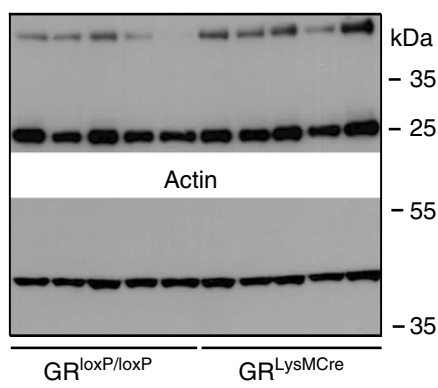

Cathepsin L
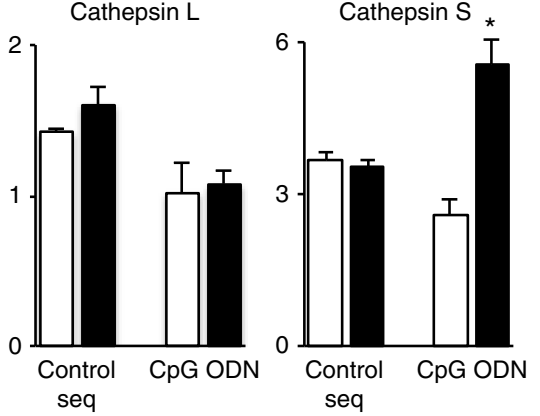

C

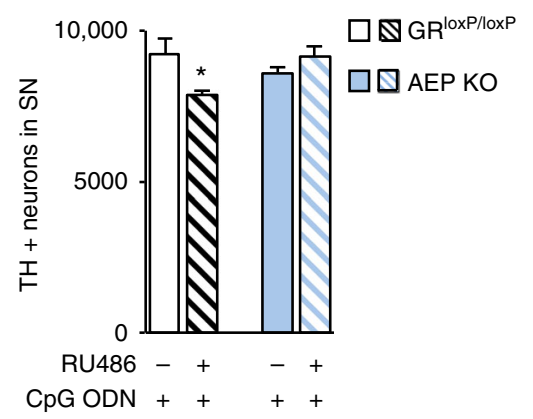

Fig. 6 In vivo assessment of the role of microglial GR on TLR9 cleavage enzymes in SN after CpG ODN injection. a RT-qPCR analysis of cathepsin B, D, K, L, and $\mathrm{S}$ mRNA levels in $\mathrm{SN}$ of control GR ${ }^{\text {loxP/loxP }}$ and GR ${ }^{\text {LysMcre }}$ mutant mice $24 \mathrm{~h}$ after intranigral injection of control sequence or $C p G$ ODN. ${ }^{*} p<0.05$ control vs. GR ${ }^{\text {LysMCre }}$ mice, $n=4-5 /$ group. $\mathbf{b}$ Western blot analysis of cathepsin B, S, and K levels in SN of control GR $R^{\text {loxP/loxP }}$ and GR $\mathrm{R}^{\mathrm{LysMcre}}$ mutant mice $48 \mathrm{~h}$ after $\mathrm{CpG}$ ODN injection. The blots were rehybridized with actin antibody. $n=5 /$ group. Full gel blots in Supplementary Fig. 4 . c Quantification of THIR neurons in $\mathrm{SN}$ of $\mathrm{GR}^{\mathrm{loxP} / \text { loxP }}$ mice and $\mathrm{AEP}^{-/-}$mice, pretreated or not with i.p. injection of $\mathrm{GR}$ antagonist RU486, 7 days after a single intranigral injection of CpG ODN. $n=5,{ }^{*} p<0.05$; pretreated with RU 486 vs. non- pretreated. All data are presented as mean with error bars as s.e.m. and Mann-Whiney non-parametric test for statistical significance

significant loss of DNs in SN. This CpG ODN-induced DN loss in GR microglial-macrophage mutants was reversed significantly by systemic administration of TLR9 antagonist COV08-0064, indicating that indeed CpG-ODN activates the TLR9 signaling pathway in SN. Of note, COV08-0064 was reported to specifically antagonize TLR9 activation peripherally; however, its action in brain was not shown. Nevertheless, its physical properties plus our results including that it is not a substrate for Pgp efflux transporter strongly indicates that it can exert effects in brain. To obtain definitive proof of its presence in CNS once injected i.p., synthesis of ${ }^{14} \mathrm{C}$-labeled version of COV08-0064 is now initiated.

The dependency of TLR9 signaling for GR was also corroborated by reducing GR activity with GR antagonist RU486 in control mice, which resulted in DN loss after intranigral CpG ODN injection. The loss of DNs in SN in microglial/macrophage GR mutants was accompanied by forelimb akinesia as revealed by cylinder test.

TLR9 is likely involved in MPTP-triggered experimental Parkinsonism. Thus, we observed a significant reduction of DN loss in TLR9-deficient mice. Our results suggest that this activation is under the control of GR as in the presence of COV08-0064 the increased death of DNs by MPTP was reversed in GR LysMcre $^{\text {L }}$ mutant mice with no effect in the control mice.

DNs are intrinsically vulnerable to an inflammatory attack ${ }^{13}$, thus, for example, intranigral injection of LPS induces specific loss of DNs through TLR4-innate immune activation of microglia $^{13}$, whereas similar low dose of LPS does not trigger death of striatal neurons ${ }^{21,46}$. In the present study, LPS-activated TLR4 and imiquimod-induced TLR7 activation induced similar DN loss in control and mutant $\mathrm{GR}^{\mathrm{LysMcre}}$ mice. The results indicating non-significant effect of microglial GR on LPS-induced DN degeneration are intriguing as we previously showed microglial GR control of neuronal death in cortical/striatal region following intra-striatal injection of $1.5 \mu \mathrm{g}$ LPS as used here ${ }^{2 \mathrm{P}}$. In addition, we also showed that microglial GR regulates inflammatory genes and NF- $\mathrm{KB}$ activation induced by LPS. The possibility of high LPS sensitivity in SN compared to striatum and cortex related to greater density of microglia was tested by reducing the dose of LPS to 0.5 and $1 \mu \mathrm{g}$. Inter-individual variability in $\mathrm{GR}^{\mathrm{LysMcre}}$ mice in response to $1 \mu \mathrm{g}$ LPS was observed suggesting that besides GR other factors control TLR4 signaling in SN microglia.

Morphological analysis of SN microglia in ipsilateral and contralateral side of SN 3 days after CpG ODN injection showed significantly greater hypertrophied microglia in $\mathrm{GR}^{\mathrm{LysMCre}}$ mutants compared to controls. In line with high microglial reactivity in GR mutants, CpG-ODN also significantly increased mRNA levels of TNF- $\alpha$, iNOS, MCP-1, MyD88 with little change in their levels in control mice. This upregulation of proinflammatory genes in the absence of microglial/macrophage GR may participate in DN loss.

In examining whether microglial GR affects TLR9 trafficking and cleavage, we uncovered unexpected actions of GR on TLR9 regulation and in microglial functions. The results of TLR9-GFP co-localization in EEA1 ${ }^{+}$early endosomes or in LAMP1 ${ }^{+}$lysosomes in control and microglial GR mutant cultures showed significant expression of TLR9-GFP in lysosomes of mutant microglia after CpG ODN treatment. CpG ODN treatment also led to increased levels of UNC93B1 and progranulin transcripts 

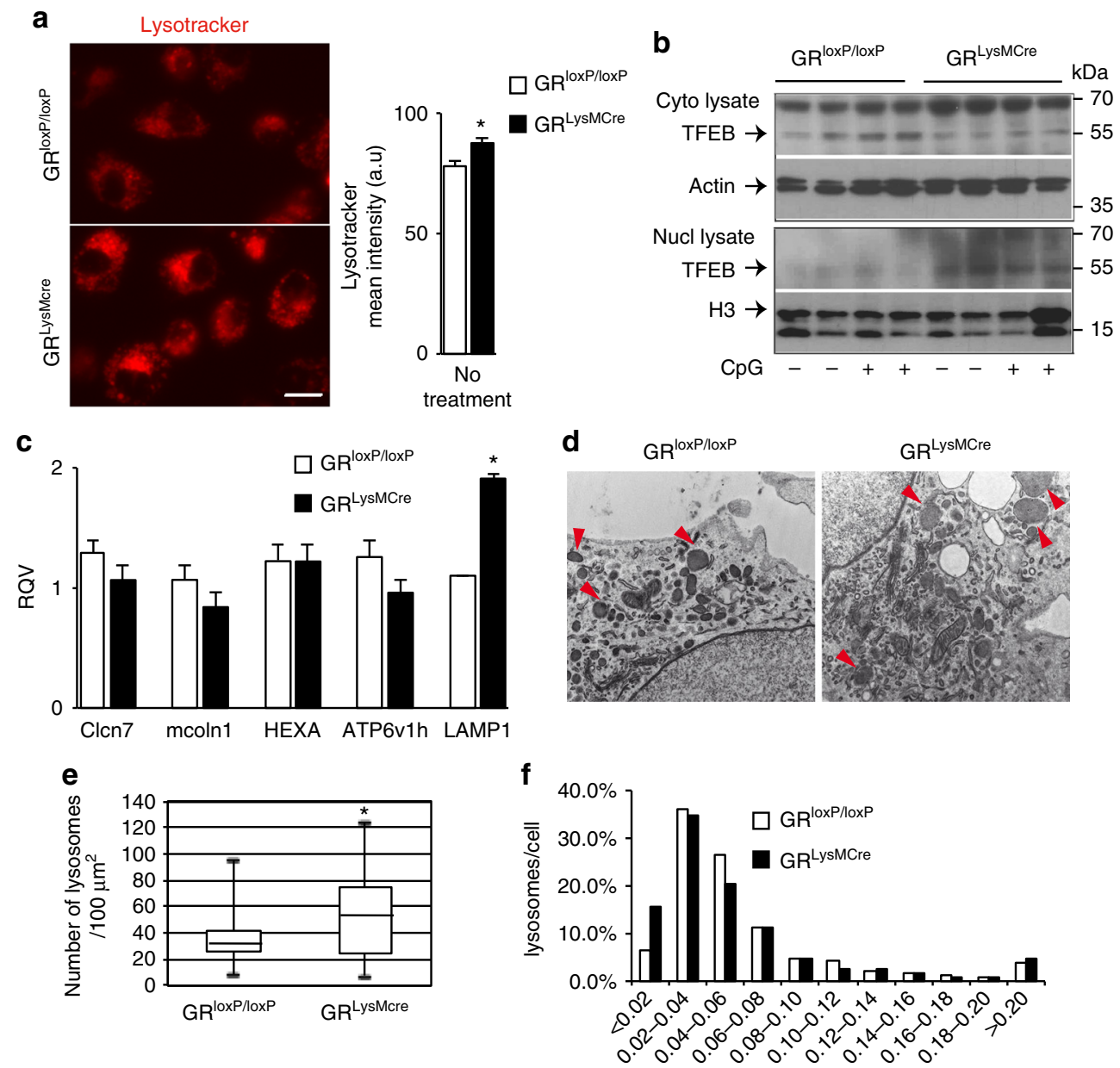

Fig. 7 Lysosomal changes in GR ${ }^{\text {LysMcre }}$ mutant microglia. a Confocal fluorescent images of Lysotracker uptake in living control and mutant primary microglial cells. Bar $=5 \mu \mathrm{m}$. The mean fluorescence intensity of Lysotracker was quantified using Image J, $n=3$ separate experiments. ${ }^{*} p<0.05$, control vs. mutant cells. b A representative western blot experiment shows an increase in nuclear fraction of TFEB in mutant microglial cultures, mirrored by a decrease in the cytoplasmic fraction of TFEB. Full gel blots in Supplementary Fig. 5. c In vitro RT-qPCR analysis of expression of lysosomal genes Clcn7, mcoln1, HEXA, ATP6v1h, and lamp1, in microglial cultures prepared from control and GR ${ }^{\text {LysMCre }}$ pups. $\beta$-microglobulin gene was used as internal control. ${ }^{\star} p<0.05$; control vs. GR ${ }^{\text {LysMCre }}$ mutant cultures, $n=3$ independent experiments. The data are presented as mean with error bars as s.e.m. and Mann-Whitney test for statistical significance. d Representative EM images of a control and a mutant cell. Red arrows point to lysosomes. e Number of lysosomes per $100 \mu \mathrm{m}^{2}$ in 21 control and 20 mutant cells. Data are expressed as Tukey boxplot where the median is indicated by a horizontal line, the bottom of the box represents the 25th percentile, the top the 75th percentile, whiskers represent minimum and maximum values. $\mathbf{f}$ Distribution of the lysosome surface $\left(\mu \mathrm{m}^{2}\right) .1386$ and 1737 lysosomes were measured, respectively, in control or mutant cells using ImageJ. $\chi^{2}$ test of independence: $\chi^{2}=84.81$ df $10, p<5.10^{-14}$

in mutant GR mice. UNC93B1 is the chaperone protein, required for exit of TLR9 from the ER to the endolysosomes ${ }^{37}$, whereas progranulin, the precursor protein of granulins, was reported as a soluble cofactor for CpG ODN binding to TLR9 ${ }^{25}$. Their high expression in mutant GR mice also argues for efficient TLR9 trafficking and processing in endolysosomes.

The major determinant for TLR9 activation is cleavage of its ectodomain near amino acid residue 477 into N-terminal and Cterminal regions by $\mathrm{pH}$-sensitive cathepsin and AEP proteases. This acid-dependent cleavage requires endosome maturation (acidification). Ligand binding to cleaved TLR9 homodimers induces a conformational change that brings the TIR domains into close proximity, enabling MyD88 recruitment ${ }^{25,37,47}$. Our results point to the involvement of AEP in TLR9 cleavage in microglia, as CpG ODN-induced DN loss after pretreatment with RU486 in AEP knock out mice was not observed compared to control mice. As the results point to TLR9 activation in mutant microglial lysosomes, we also examined alterations in microglial lysosomes in the absence of GR. We observed increased
Lysotracker fluorescence intensity in GR mutant microglia. Further characterization by transmission EM showed enhanced number and altered size distribution of lysosomes compared to control microglia. Increased level of TFEB transcription factor in nuclear fraction together with augmented expression of LAMP1 in GR mutant microglia imply a change in lysosomal biogenesis and function. Overall, these results show that microglial GR regulates TLR9 trafficking and lysosomal environment.

In our search for relevant TLR9 activators in PD and given that unmethylated CpG sequences are present in mitochondrial DNA, we examined whether mitochondrial DNA can induce dopamine neurodegeneration through TLR9 activation. The role of CpGrich mitochondrial DNA in triggering inflammation through TLR9 has been previously demonstrated. Oka et al. ${ }^{43}$ showed that mitochondrial DNA that escapes from autophagy-mediated degradation leads to TLR9-mediated inflammatory responses in cardiomyocytes and causes inflammation and heart failure. In systemic lupus erythematous, recognition of self-nucleic acids by TLR7 and TLR9 with ensuing innate inflammation is an 
a

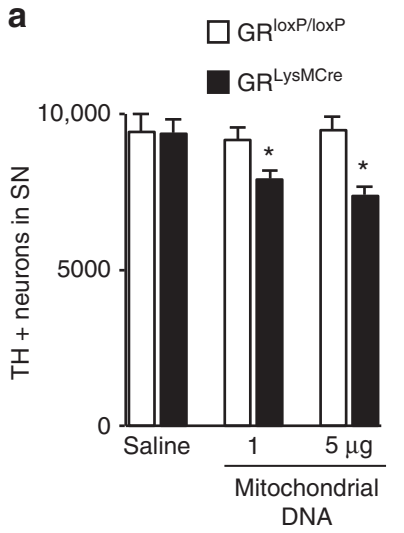

b

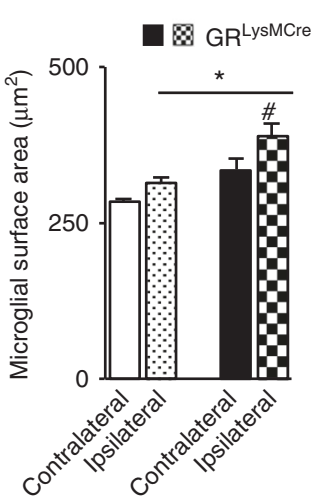

C

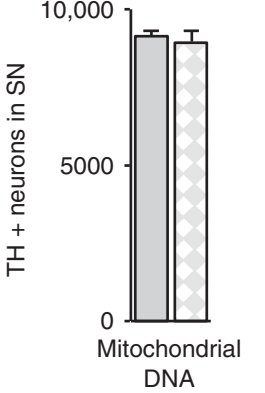

TLR9 KO

TLR9 KO- RU486

Fig. 8 DN loss triggered by mitochondrial DNA in GR ${ }^{\text {LysMcre }}$ mutants. a TH-IR neurons in SN of GR ${ }^{\text {loxP/loxP }}$ mice and GR ${ }^{\text {LysMCre }}$ mice quantified 7 days after a single intranigral injection of saline or 1 or $5 \mu g \mu l^{-1}$ mitochondrial DNA. ${ }^{\star} p<0.05$; GR ${ }^{\text {loxP/loxP }}$ mice and GR ${ }^{\text {LysMCre }}$ mice. $n=5 /$ group. $\mathbf{b}$ Microglial surface area, an index of microglial hypertrophy, was analyzed by ImageJ in same mice as $\mathbf{a}$. The results show increased microglial hypertrophy in GR ${ }^{L y s M C r e}$ mutant mice compared to controls ${ }^{*} p<0.05$ control vs. mutant; $\# p<0.05$ ipsilateral vs. contralateral side. c Quantification of TH-IR neurons in SN of TLR9 $-/$ - mice, pretreated or not with i.p. injection of GR antagonist RU486, 7 days after a single intranigral injection of $1 \mu \mathrm{g}$ of mitochondrial DNA. $n=5 /$ group. All data are mean with error bars as s.e.m. and Mann-Whitney non-paramtetric test for significance

important pathological feature, and, interestingly is correlated with reduced GC activity ${ }^{48}$. We found $16-20 \%$ DN loss with either 1 or $5 \mu \mathrm{g}$ mitochondrial DNA which was far less than with CpG ODN. The CpG copy numbers of these two TLR9 ligands are very different. Thus, $1 \mu \mathrm{g} C \mathrm{Cg}$ ODN can be estimated to have approximately $4.6 \times 10^{14}$ copies of $\mathrm{CpG}$, whereas similar concentration of mitochondrial DNA has around $1.3 \times 10^{4}$ copies taking into account $850 \mathrm{CpG}$ sites in $16.57 \mathrm{kbp}$ of mitochondrial DNA $^{49}$. This DN loss was GR and TLR9-dependent as it occurred only in GR LysMCre $^{\text {mice and not in TLR9 }}{ }^{-1-}$ mice treated with RU486. The signals that can activate microglia in SN include oligomeric and fibrillar forms of a-synuclein, neuromelanin, and HMGB1 released from dying $\mathrm{DNs}^{50-53}$. Under normal circumstances, self-nucleic acids, which are methylated, are degraded before reaching the endolysosomes where TLR3, TLR7, and 9 are localized. However, if nucleotides are in complexes with HMGB1 released by dying cells, they resist degradation. Recently, high levels of HMGB1 in CSF and serum of PD patients were reported $^{53}$. As well, PD patients harboring G2019S LRRK2 mutation were reported to have a high concentration of mitochondrial DNA in CSF; however, the source of mitochondrial DNA remain unknown ${ }^{54}$. Other possible situations, which may sensitize microglial TLR9 pathway, include CNS and systemic infections as well as brain trauma. Indeed, DNA viruses (known to activate TLR9 $^{55}$ ) such as Herpes simplex virus, Epstein-Barr virus, Cytomegalovirus, and Varicella zoster virus have been associated to Parkinsonism ${ }^{56}$.

Regarding GCs and GR in PD, several studies have reported significantly high circulating levels of cortisol in PD patients ${ }^{16,57,58}$. Chronically high GC levels are known to compromise GR activity in immune cells ${ }^{59}$, which could permit TLR9 induction. Indeed our present work argues for a decreased GR transcriptional activity in microglia. As schematized in Fig. 9, overall, our results suggest that decreased microglial GR activity in SN can lead to an enhancement of dopamine neurodegeneration and pathogenesis in PD patients. Specifically, this loss of GR enables activation of microglial TLR9 inflammatory pathway, which can play a role in the progression of $\mathrm{PD}$ pathology.

\section{Methods}

Human SN autopsy samples. The human post-mortem samples were obtained from brains collected in a Brain Donation Program of the Brain Bank "NeuroCEB" run by a consortium of Patients Associations that include ARSEP (Association for
Research on Multiple Sclerosis), CSC (cerebellar ataxias), and France Parkinson. The signed consents were either from the patients or their next of kin in their name, in accordance with the French Bioethical Laws.

SN sections for IF experiments were from three males, one female control subjects: age $75.25 \pm 7.4$ years, post-mortem delay: $23.87 \pm 13.5 \mathrm{~h}$; and from three males, one female PD patients: age $79 \pm 2.3$ years, post-mortem delay: $30 \pm 12.9 \mathrm{~h}$ for PD patients. SN western blot samples were from three male control subjects: age $68 \pm 10$ years, post-mortem delay: $11.5 \pm 9.96 \mathrm{~h}$; and two males, one female PD patients: age $78 \pm 5.2$ years, post-mortem delay: mean $39.5 \mathrm{~h}$ for two and one not determined. All values are mean \pm s.e.m. Two of the samples in IF and western blot experiments were from same PD patients.

Mouse models. The Nr3C1loxp/loxp;LysMCre mice (thereafter denominated $\mathrm{GR}^{\text {LysMCre }}$ ) were generated by crossing Nr3C1loxp mice with LysMCre transgenic mice, which express Cre recombinase inserted by homologous recombination under the lysozyme $\mathrm{M}$ promoter gene $\mathrm{e}^{22,60}$. The mice were backcrossed to C57BL/6 for at least 10 generations. Generation of DATicre (Tg BAC-DATiCrefto) and $\mathrm{GR}^{\mathrm{DATiCre}}$ mice inactivated for GR gene in DNs is described in ref. ${ }^{30}$. $\mathrm{AEP}^{-/-}$ mice were generated in C. Peters' lab (Freiburg) and backcrossed 11 times on B6 background. TLR9 KO mice were obtained from Drs. N. Doyen and C. Cousin, Pasteur Institute, Paris, and also from Dr. L. Alexopoulou, Centre d'Immunologie de Marseille-Luminy. GR ${ }^{\mathrm{CX} 3 \mathrm{CR} 1 \mathrm{CreER} 2}$ mice were generated by crossing CX3CR1CreER2 transgenic mice ${ }^{23}$ with $N r 3$ C1loxp mice. For Cre recombination in GR ${ }^{\mathrm{CX} 3 \mathrm{CR} 1 \mathrm{CreER} 2}$ mice, both control and GR ${ }^{\mathrm{CX} 3 \mathrm{CR} 1 \mathrm{CreER} 2}$ were injected i.p. with $2 \mathrm{mg}$ of tamoxifen (Sigma) per day for 5 days and experiments undertaken 3 weeks after to ensure the disappearance of GR only in CNS microglia $^{23}$.

Mice were group-housed under a controlled photo-period (12-h day-night cycles), at constant room temperature $\left(22^{\circ} \mathrm{C}\right)$ and had access to food and water ad libitum. GR ${ }^{\text {LysMCre }}, G^{\text {DATiCre }}, G R^{\text {CX3CR1CreER2 }}$ mice were genotyped for the presence of Nr3C1loxp allele and Cre or iCre transgene by PCR. All studies were performed in accordance with ethical guidelines of European Convention for the protection of Vertebrate Animals (directives of 2013: 2010/63/EU) and experimental protocol approval from French ministry of Research. Control and mutant littermate mice were randomly grouped for treatment.

Stereotaxic injections. For stereotaxic surgery, 3-5-months-old GR ${ }^{\text {DATiCre }}$, $\mathrm{GR}^{\mathrm{LysMCre}}, \mathrm{GR}^{\mathrm{CX} 3 \mathrm{CR} 1 \mathrm{CreER} 2}$ mutant male mice and $\mathrm{GR}^{\text {loxp/loxp }}$ control littermates were anesthetized by an i.p. injection of $100 \mathrm{mg} \mathrm{kg}^{-1} \mathrm{ketamine}$ and $10 \mathrm{mg} \mathrm{kg}^{-1}$ xylazine. The scalp was shaved and a small hole was made at the surface for injection into the left substantia nigra using the following stereotaxic coordinates in mouse Paxinos atlas (David Kopf Instruments, Tujunga, CA, USA) from Bregma $-2.8 \mathrm{~mm}$ anteroposterior, $+1.3 \mathrm{~mm}$ mediolateral, and $-4 \mathrm{~mm}$ dorsoventral. In all, $0.5,1$, and $1.5 \mu \mathrm{g}$ of TLR4 agonist LPS (LPS Escherichia coli, serotype 055:B5; Sigma, St. Quentin Fallavier, France), $1 \mu \mathrm{g}$ of TLR2 agonist (Pam3Cys-Ser- (Lys) 4 Trihydrochloride; Abcam, ab142085), $1 \mu \mathrm{g}$ of TLR7 agonist, Imiquimod (Enzo Life Sciences, ALX-420-039-M100), $0.96 \mu \mathrm{g}(1 \mu \mathrm{M})$ of TLR9 agonist ODN 1826 (TLRgradeTM; 5'-TCCATGACpGTTCCTGACpGTT-3') (Enzo Life Sciences, ALX-746-002-T100) or a control ODN sequence (5'-TCCATGAGCTTCCTGAGCTT- $3^{\prime}$ ) dissolved in PBS were injected using $10 \mu \mathrm{l}$ Hamilton syringe into the substantia nigra over a 5-min period. For Adeno-associated vector (AAV) serotype 6 PGK-A53T- $\alpha$-synuclein injections, the stock of $1.97 \times 10^{13} \mathrm{gp}$ (genomic particles/ 


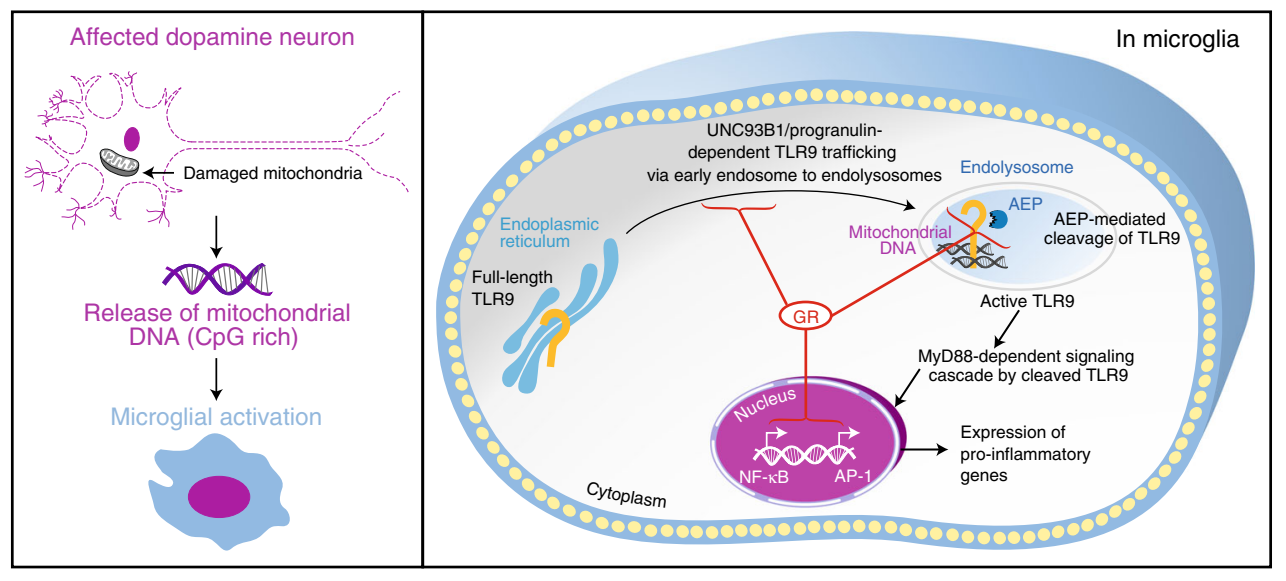

Fig. 9 GR regulation of TLR9 activation in microglia that impacts dopamine neuron death. In Parkinson's disease, chronically high cortisol levels likely compromise GR activity in innate immune cells. TLR9 levels are modulated in post-mortem Parkinson disease brains. Reduced GR activity in microglia permits activation of TLR9 by DAMPS such as CpG-rich mitochondrial DNA released from affected dopamine neurons that selectively exacerbates dopamine neuronal loss in substantia nigra. In microglia, intact GR activity acts as a brake for TLR9 activation and downstream inflammatory gene expression by regulating TLR9 trafficking through expression of UNC93B1 and progranulin and AEP-mediated cleavage of TLR9

$\mathrm{ml}$ ) was diluted to $2.5 \times 10^{9} \mathrm{gp} \mu \mathrm{l}^{-1}$ in saline. $2 \mu \mathrm{l}$ of AAV-PGK-A53T $\alpha$-synuclein was injected and mice sacrificed after 8 weeks.

Intraperitoneal RU486 pretreatment. $\mathrm{GR}^{\mathrm{LysMCre}}$ mutants, control $\mathrm{GR}^{\text {loxp/loxP, }}$, AEP KO, and TLR9 KO mice were pretreated by an IP injection of $30 \mathrm{mg} \mathrm{kg}^{-1}$ RU486 dissolved in 1:9 ethanol:oil $16 \mathrm{~h}$ before stereotaxic injection of CpG ODN.

MPTP and TLR9 antagonist COV08-0064 treatment. Experiments were performed on 3-5-months-old TLR9 k.o. and GR ${ }^{\mathrm{LySMCre}}$ mutant mice and their control littermate male mice. For TLR9 k.o. experiment the mice received four i.p. injections of $20 \mathrm{mg} \mathrm{kg}^{-1} \mathrm{MPTP} . \mathrm{HCl}$ or the same volume of saline at $2 \mathrm{~h}$ intervals. Mice were sacrificed 7 days after the last injection. For MPTP-COV08-0064 experiment, the $\mathrm{GR}^{\operatorname{loxp} / \text { loxP }}$ and $\mathrm{GR}^{\mathrm{LysMCre}}$ mutant mice were injected with $120 \mathrm{mg}$ $\mathrm{kg}^{-1}$ COV08-0064 (dissolved in saline) $1 \mathrm{~h}$ prior to injection of $18 \mathrm{mg} \mathrm{kg}^{-1}$ MPTP$\mathrm{HCl}$. MPTP dose was reduced as increased lethality was observed by combination of COV08-0064-MPTP treatment particularly in $\mathrm{GR}^{\mathrm{LysMCre}}$ mutant mice during injection paradigm. $24 \mathrm{~h}$ after injections, both control and mutant mice recovered in similar manner. For MPTPv + COV08-0064 group, the mice were injected with the same concentration of COV08-0064 $\times 1$ for 2 consecutive days following MPTP treatment and sacrificed 4 days later.

Behavior tests. For cylinder test, mice were placed individually inside a glass cylinder (diameter $15 \mathrm{~cm}$, height $30 \mathrm{~cm}$ ). A camera was placed above the cylinder to allow full vision of the cylinder. The session was videotaped during $5 \mathrm{~min}$ for later scoring. No habituation of the mice to the cylinder was made. The assessment was adapted from previous study ${ }^{32}$ : the first 20 wall touches with the ipsilateral and the contralateral forelimbs (contacts with fully extended digits), executed independently, were counted. Simultaneous paw touches were excluded from the analysis. Data are expressed as a percentage of contralateral touches, calculated as (contralateral touches)/(ipsilateral touches + contralateral touches) $\times 100$. The analysis performed by the experimenter blinded to the genotype of mice.

For Rotarod test, mice were first habituated to stay on the rotarod apparatus (LSI Letica Scientific Instrument-Rota-Rod LRS) at $5 \mathrm{rpm}$ for 1 day, followed by habituation the next 2 days on the accelerating rotarod with rotarod speed linearly increasing from 5 to 40 r.p.m. over 300 s, so that the latency to fall for all was 3-5 $\mathrm{min}$. One week later the animals were tested on accelerating rotarod three trials/ day, at least $10 \mathrm{~min}$ interval for 3 days. The latency to fall from the rod was recorded for each trial. Mice remaining on the rod for more than $300 \mathrm{~s}$ were removed and their time scored as $300 \mathrm{~s}$. The mice were injected with CpG-ODN the following day and test repeated 1 week later.

IF and western blot in human brain. Midbrain sections were verified for decrease in TH-IR in PD samples and localization of SN. IF of GR and Ibal was carried out on $30 \mu \mathrm{m}$ fresh sections ( $n=4$ for control subjects and 3 for PD patients) and with $10 \mu \mathrm{m}$ paraffin sections $(n=1 \mathrm{PD})$. As similar results were obtained with sections from fresh-frozen tissues and paraffin-embedded sections, the results were pooled. The method of Monier et al. ${ }^{61}$ was used to achieve Ibal labeling. Paraffinembedded sections were deparaffinated in a series of xylene/alcohol solutions, and antigen retrieval was undertaken by heating the sections at $80^{\circ} \mathrm{C}$ for $20 \mathrm{~min}$ in 10 $\mathrm{mM}$ sodium citrate buffer, $\mathrm{pH}$ 6.0. Cryostat fresh sections were air-dried before fixation with freshly prepared 4\% PFA in PBS solution for $20 \mathrm{~min}$. The sections were rinsed in Tris-buffered saline (TBS) solution containing $0.5 \%$ Triton before blocking for $2 \mathrm{~h}$ in $2 \%$ goat serum in TBS- $0.5 \%$ Triton. They were incubated with rabbit polyclonal anti-Ibal antibody (WAKO-1/200) and mouse anti-GR antibody (AbCam-1/200) in $2 \%$ goat serum/TBS- $0.5 \%$ Triton for $48 \mathrm{~h}$ at $4{ }^{\circ} \mathrm{C}$. After washes in TBS- $0.5 \%$ Triton, they were incubated with secondary antibodies: Alexa goat anti-rabbit 488 and donkey anti-mouse Cy3, washed, stained with Hoechst 33342 and mounted using vectashield.

For western blot analysis, SN was dissected and protein homogenates were prepared as reported previously ${ }^{16}$ in lysis buffer containing $62.5 \mathrm{mM}$ Tris $\mathrm{pH} 6.8$, $1 \%$ SDS, $10 \%$ glycerol, $5 \%$ beta-mercaptoethanol, and protease inhibitors (complete cocktail, Roche). $20 \mu \mathrm{g}$ lysates were loaded onto $10 \%$ acrylamide gel. After transfer onto a PVDF membrane, the blots were probed with TLR9 primary antibody (1/ 500 Imgenex $)^{16}$. The same blot was re-incubated with actin for loading control. After incubation with secondary peroxidase-conjugated antibodies (GE Healthcare) diluted at 1:2000, signals were visualized by using ECL detection kit (GE Healthcare).

Immunohistochemistry and western blot in mice. For immunohistochemistry, mice were anesthetized with $\mathrm{CO}_{2}$ and rapidly perfused transcardially with ice-cold $0.1 \mathrm{M}$ sodium phosphate buffer (PBS) followed by ice-cold $4 \%$ paraformaldehyde (PFA) in $0.1 \mathrm{M}$ sodium PBS. Brains were rapidly removed from the skull and postfixed for $24 \mathrm{~h}$ in fresh $4 \%$ PFA in PBS solution. $30 \mu \mathrm{m}$ thick coronal sections were cut serially as described in Ros Bernal et al. ${ }^{11}$ and stored in PBS containing $0.4 \%$ sodium azide at $4{ }^{\circ} \mathrm{C}$ until use. Sections of substantia nigra, at $180 \mu \mathrm{m}$ interval, were rinsed in PBS, treated with $0.3 \% \mathrm{H}_{2} \mathrm{O}_{2} / \mathrm{PBS}$ for $15 \mathrm{~min}$ and non-specific $\mathrm{Fc}$ binding sites were blocked in $2 \%$ newborn goat serum (Sigma-Aldrich) in PBS-T (PBS/0.1\% Triton X-100); sections were incubated with rabbit polyclonal anti-Ibal (W1W019-1974, 1:750; Wako Chemicals), or mouse monoclonal anti-TH (MAB 318, 1:1000, Millipore), anti PV25 (1:500, MAB 1572, Sigma) primary antibodies for $24 \mathrm{~h}\left(\right.$ at $\left.4{ }^{\circ} \mathrm{C}\right)$, with constant shaking. After three washes, sections were incubated for $2 \mathrm{~h}$ with appropriate anti-mouse or anti-rabbit biotinylated secondary antibodies (1:500; Vector Laboratories). Binding of antibody was detected with avidin-biotin peroxidase $\mathrm{ABC}$ kit according to manufacturer's instructions (Vectastain, Vector Labs) using the chromogen diaminobenzidine (Sigma) as a substrate for the peroxidase. Sections were mounted on superfrost plus slides and dehydrated in graded ethanol series and xylene and then coverslipped. Fluoro-Jade B staining procedure was carried out according to the manufacturer's instructions (HistoChem Inc., Jefferson, AR, USA).

For western blot analysis of cathepsins, protein lysates were prepared in the lysate buffer as described above from punches of ipsilateral SN of control and $\mathrm{GR}^{\mathrm{LysM} \text { cre }}$ mice injected with CpG ODN. $25 \mu \mathrm{g}$ lysates were loaded on $14 \%$ acrylamide gels and after transfer onto a PVDF membrane, the blots were probed at 1/250 with mouse monoclonal anti-cathepsin B (H-5, SC36558 Santa Cruz), rabbit polyclonal anti-cathepsin $\mathrm{K}$ (ab85716, AbCam) and mouse monoclonal anticathepsin S (E-3 SC271619 Santa Cruz) and actin used as loading control.

Image analysis. Quantification of DN loss was performed stereologically as described previously ${ }^{16}$ on regularly spaced DAB sections of mesencephalon covering the whole SN (from rostral pole of the SN to the locus coeruleus) by brightfield microscopy using a Nikon microscope (Eclipse, $20 \times$ objective) equipped with a semi-automatic stereology system (Mercator software; Explora Nova VisioScan T4.18 system). The genotype of mice was unknown to the investigator at the time of quantification. 
Fluoro-Jade B-positive cells were quantified from photomicrographs taken using FITC filter at $4 \times$ objective (Nikon). For all quantifications related to activated microglia, DAB-labeled sections were used. ImageJ software (NIH, USA) was used for quantification of Fluoro- Jade B-positive neurons, microglial densities, as well as microglial surface area.

For human brain sections, stacks of consecutive images at $2 \mu \mathrm{m}$ intervals were acquired sequentially using $40 \times$ objective on a confocal laser-scanning Leica microscope. Three to seven fields were selected at random in SN per section then reconstructed and quantified using ImageJ software.

RT-quantitative PCR. Mice were euthanized $24 \mathrm{~h}$ after $\mathrm{CpG}$ injection and their brains snap frozen in isopentane at $-25^{\circ} \mathrm{C}$. The ipsilateral lesioned and contralateral non-lesioned SN regions were rapidly dissected by punch at -5 to $-10^{\circ} \mathrm{C}$. Total RNA was prepared using the RNeasy lipid mini kit (Qiagen) in sterile conditions. The RNA integrity and concentration was determined by using Nanodrop.

In all, $1 \mu \mathrm{g}$ of total RNA from whole tissue and $500 \mathrm{ng}$ from cell cultures were used for cDNA synthesis with Superscript III (Invitrogen, St. Aubin, France). HPRT and $\beta$-microglobulin were used as internal controls for whole tissues and in vitro microglial cultures, respectively. qPCR experiments were carried out on Roche Light Cycler 480-II (Roche Diagnostic) using Syber Green master mix.

\section{In vitro experiments on primary microglial cells from P1 control and}

$\mathbf{G R}^{\text {LysMCre }}$ pups. Cerebral hemispheres were dissected and cortices extracted from newborn mice after removal of meninges. After dissociation and homogenization, cells were seeded at 30,000 cells ml ${ }^{-1}$ in DMEM containing $10 \%$ heat-inactivated FCS on poly-ornithine plated dishes. Medium was changed at days 2 and 4, and microglial cells were collected at day 12 by shaking culture dishes to detach cells adhering to the astrocyte monolayer, as described.

Freshly collected microglia were seeded on 24-well plates in DMEM containing $4 \%$ FCS. They were treated $48 \mathrm{~h}$ later with, $1 \mu \mathrm{m}$ CpG ODN for $1 \mathrm{~h}$, in DMEM medium containing $0.5 \%$ FCS. Microglial cells were collected in RLT buffer from RNeasy Mini Kit (Qiagen) and stored at $-80^{\circ} \mathrm{C}$ for total RNA extraction.

Transfection with TLR9-GFP plasmid. $10^{6}$ microglial cells harvested were transfected with $1 \mu \mathrm{g}$ of cDNA coding for TLR9-GFP ${ }^{38}$ using Amaxa Mouse macrophage nucleofector kit (Lonza), according to the manufacturer's instructions. $3 \mathrm{~h}$ later, when the cells had adhered, the medium was changed to: DMEM containing 4\% FCS, $2 \mathrm{mM}$ glutamine (Gibco 25030-024), $50 \mu \mathrm{m}$ 2mercaptoethanol, GM-CSF $0.1 \mu \mathrm{g} \mathrm{ml}^{-1}$ (R\&D Research 415). $24 \mathrm{~h}$ later, cells were stimulated with TLR9 agonist, CpG ODN

IF of microglia in vitro. Autofluorescence was quenched with ammonium chloride/PBS. Non-specific Fc binding sites were blocked with $0.25 \%$ gelatin in PBS. Primary antibodies against EEA1 (610457 Brand BD Transduction Laboratories) and LAMP1 (553792BD pharmingen) were incubated overnight at $4{ }^{\circ} \mathrm{C}$. Secondary antibodies coupled to cy3, Alexa 488, cy5 (Invitrogen) were used at 1/400 dilution for $2 \mathrm{~h}$ followed by $15 \mathrm{~min}$ in a PBS solution containing $4^{\prime}, 6^{\prime}$-diamidino-2-phenylindole (DAPI) (1/2000) and then mounted for examination under a fluorescence microscope.

Quantification of co-localization. Stacks of consecutive images at $0.15 \mu \mathrm{m}$ intervals were acquired sequentially with three lasers at $488 \mathrm{~nm}$ (TLR9-GFP) and 555 $\mathrm{nm}$ (EEA1) and $633 \mathrm{~nm}$ (LAMP1) at objective (63×) on a confocal laser-scanning Leica microscope. Confocal fluorescent images were converted to binary images in ImageJ. The fluorescence across one entire image was determined; a threshold for positive staining was set for each channel (green, red, or far red). The percentage of co-localization between two given channels was derived from the number of positive pixels common to these two channels (TLR9 GFP and LAMP1; or TLR9 GFP and EEAl, for example), divided by the number of positive pixels within the TLR9-GFP image.

Lysotracker treatment and image acquisition. Microglial cells were plated in 8well ibi-treated (IBIDI-optimal cell adhesion) chamber followed by treatment with $60 \mathrm{nM}$ lysotracker red (DND 99 Invitrogen) in phenol-free 0.5\% FCS DMEM for $30 \mathrm{~min}$, in a thermostated chamber at $37^{\circ} \mathrm{C}$ and $\mathrm{CO}_{2}$. Images of living cells were taken with the confocal microscope $63 \times$ objective using the $577 \mathrm{~nm}$ filter.

Nuclear and cytoplasmic fractionation and immunoblots. Cells were trypsinized at $37^{\circ} \mathrm{C}$ for $5-10 \mathrm{~min}$. Reaction was stopped by adding DMEM containing $10 \%$ FCS, then harvested and centrifuged at $1300 \mathrm{rpm}$ for $20 \mathrm{~min}$ at $4{ }^{\circ} \mathrm{C}$. Pellets were then resuspended in $100 \mu \mathrm{l}$ Buffer A (10 mM HEPES-KOH pH 7.9, $1.5 \mathrm{mM} \mathrm{MgCl}$, $10 \mathrm{mM} \mathrm{KCl}, 0.5 \mathrm{mM}$ DTT, $0.2 \mathrm{mM}$ PMSF with protease inhibitors), allowed to swell on ice for $10 \mathrm{~min}$, vortexed then centrifuged for $10 \mathrm{~s}$. Supernatant containing cytoplasmic proteins was recuperated and stored at $-80^{\circ} \mathrm{C}$. Pellets were resuspended in $100 \mu$ RIPA buffer ( $150 \mathrm{mM} \mathrm{KCl}, 50 \mathrm{mM}$ HEPES, $0.5 \% \mathrm{NP} 40,0.2 \% \mathrm{Na}$ deoxycholate, $0.5 \mathrm{mM}$ EGTA, $0.5 \mathrm{mM}$ EDTA, with protease inhibitors), centrifuged at $12,000 \mathrm{rpm}$ for $15 \mathrm{~min}$ at $4^{\circ} \mathrm{C}$. Supernatant containing nuclear proteins was recuperated and stored at $-80^{\circ} \mathrm{C} .20 \mu \mathrm{g}$ of protein samples were loaded on Novex
NuPage 4-12\% Bis-Tris gradient gels (Invitrogen). After transfer onto a PVDF membrane, the blots were probed with TFEB primary antibody (SAB4503154, Sigma). The same blots were re-incubated with either actin or histone $\mathrm{H} 3$ antibodies for loading control. After incubation with secondary peroxidase-conjugated antibodies (GE Healthcare) diluted at 1:2000, signals were visualized by using ECL detection kit (GE Healthcare).

Transmission electron microscopy. Primary microglial cells were fixed in $2 \%$ glutaraldehyde (Agar) diluted in 0.1 M PBS at pH 7.4. Cells were washed and incubated for $1 \mathrm{~h}$ in $1 \%$ osmium tetroxide (EMS) reduced with $1.5 \%$ potassium ferrocyanide diluted in 0.1 M sodium cacodylate (EMS). Cells were dehydrated through graded concentration of ethanol (50-70-96-100\%) and infiltrated in epoxy resin (Agar 100 resin kit, Agar) at room temperature according to the manufacturer's instructions and polymerized for $48 \mathrm{~h}$ at $60^{\circ} \mathrm{C}$. Ultrathin sections $(70$ $\mathrm{nm}$ ) were cut with an ultramicrotome (Ultracut, Leica Microsystems) and collected on 200 Mesh copper grids (Agar). Sections were stained with 2.5\% aqueous uranyl acetate (Prolabo) and $1 \%$ lead citrate (Agar) before observation at $80 \mathrm{kV}$ with a Zeiss 912 Omega TEM, equipped with side-mounted $2 \mathrm{k} \times 2 \mathrm{k}$ Veleta (Olympus) CCD camera controlled with iTEM (Olympus) software. Images were analyzed using ImageJ. The number and surface of individual lysosomes per $\mu \mathrm{m}^{2}$ were calculated in 21 control cells (1386 lysosomes) and 20 mutant cells (1787 lysosomes). After counting, distributions of lysosome surface were compared with the $\chi^{2}$ test of independence, according to the formula in Eq. (1), where $A_{i j}$ and $E_{i j}$ refers, respectively, to the observed and the theoretical values:

$$
X^{2}=\sum \sum \frac{\left(\left(A_{i j}\right)-E_{i j}\right)^{2}}{E_{i j}} .
$$

Mitochondrial DNA preparation. Mitochondria were isolated from the brains and livers of C57Bl6 mice using "mitochondria isolation kit for tissue" (Thermo Scientific), according to the manufacturer's protocol. Mitochondrial DNA was extracted from the mitochondrial pellet according to the procedure described by Palva and Palva ${ }^{62}$. Briefly, mitochondrial pellet was resuspended in $200 \mu \mathrm{l}$ buffer containing $50 \mathrm{mM}$ glucose, $10 \mathrm{mM}$ EDTA, and $25 \mathrm{mM}$ Tris- $\mathrm{HCl}(\mathrm{pH}$ 8) on ice. To lyse the mitochondria and denature the contaminating nuclear DNA, $400 \mu \mathrm{l}$ alkaline SDS solution $(0.2 \mathrm{M} \mathrm{NaOH}, 1 \%$ SDS $)$ was added and the suspension thoroughly mixed. After $5 \mathrm{~min}$ incubation on ice, $300 \mu \mathrm{l}$ of $3 \mathrm{M}$ potassium acetate was added, and the contents of the tube gently mixed. The tube was maintained at $-70{ }^{\circ} \mathrm{C}$ for $2 \mathrm{~min}$ and centrifuged for $10 \mathrm{~min}$ at $10,000 \times g .750 \mu \mathrm{l}$ of the clear supernatant was removed and the mtDNA precipitated by adding $450 \mu \mathrm{l}$ isopropanol and holding the tube for $5 \mathrm{~min}$ at $-70^{\circ} \mathrm{C}$.

The precipitate was collected by centrifugation, washed with $70 \%$ ethanol and and the pellet stored at $-20^{\circ} \mathrm{C}$. The mtDNA pellet was resuspended in $100 \mu \mathrm{l}$ of 10 $\mathrm{mM}$ Tris- $\mathrm{HCl}(\mathrm{pH} 8)$ and $1 \mathrm{mM}$ EDTA, treated with DNase-free RNase, and phenol extracted. DNA was re-precipitated with ethanol in the presence of $150 \mathrm{mM}$ $\mathrm{NaCl}$ overnight at $-80^{\circ} \mathrm{C}$. The precipitate was washed with $70 \%$ ethanol, dried, and resuspended in $15 \mu \mathrm{l}$ of $10 \mathrm{mM}$ Tris- $\mathrm{Cl}(\mathrm{pH} 8)$ and $1 \mathrm{mM}$ EDTA.

Statistical analysis. Data are expressed as mean \pm s.e.m. Statistical analysis was performed using Mann-Whitney tests unless otherwise stated. For animal and human studies, $n=5$ for most data points, or 3-4 as stated. Differences of $p<0.05$ were considered statistically significant. Statistical analyses were carried out using StatView 5.0 software.

Data availability. The data supporting the finding in this study are available from corresponding author upon request.

Received: 4 July 2016 Accepted: 10 May 2018

Published online: 22 June 2018

\section{References}

1. Hirsch, E. C. \& Hunot, S. Neuroinflammation in Parkinson's disease: a target for neuroprotection? Lancet Neurol. 8, 382-397 (2009).

2. Halliday, G. M. \& Stevens, C. H. Glia: initiators and progressors of pathology in Parkinson's disease. Mov. Disord. 26, 6-17 (2011).

3. Herrero, M. T., Estrada, C., Maatouk, L. \& Vyas, S. Inflammation in Parkinson's disease: role of glucocorticoids. Front. Neuroanat. 9, 32 (2015).

4. Jenner, P. \& Olanow, C. W. The pathogenesis of cell death in Parkinson's disease. Neurology 66, S24-S36 (2006).

5. Lawson, L. J., Perry, V. H., Dri, P. \& Gordon, S. Heterogeneity in the distribution and morphology of microglia in the normal adult mouse brain. Neuroscience 39, 151-170 (1990).

6. Kim, W. G. et al. Regional difference in susceptibility to lipopolysaccharideinduced neurotoxicity in the rat brain: role of microglia. J. Neurosci. 20, 6309-6316 (2000). 
7. Ransohoff, R. M. \& Brown, M. A. Innate immunity in the central nervous system. J. Clin. Invest. 122, 1164-1171 (2012).

8. Barton, G. M. A calculated response: control of inflammation by the innate immune system. J. Clin. Invest. 118, 413-420 (2008).

9. Saijo, K. \& Glass, C. K. Microglial cell origin and phenotypes in health and disease. Nat. Rev. Immunol. 11, 775-787 (2011).

10. Takeuchi, O. \& Akira, S. Pattern recognition receptors and inflammation. Cell 140, 805-820 (2010).

11. Ghosh, A. et al. Selective inhibition of NF-kappaB activation prevents dopaminergic neuronal loss in a mouse model of Parkinson's disease. Proc. Natl Acad. Sci. USA 104, 18754-18759 (2007).

12. Hunot, S. et al. JNK-mediated induction of cyclooxygenase 2 is required for neurodegeneration in a mouse model of Parkinson's disease. Proc. Natl Acad. Sci. USA 101, 665-670 (2004).

13. Castano, A., Herrera, A. J., Cano, J. \& Machado, A. Lipopolysaccharide intranigral injection induces inflammatory reaction and damage in nigrostriatal dopaminergic system. J. Neurochem. 70, 1584-1592 (1998).

14. Deleidi, M. \& Gasser, T. The role of inflammation in sporadic and familial Parkinson's disease. Cell. Mol. Life Sci. 70, 4259-4273 (2013).

15. Kim, C. et al. Neuron-released oligomeric alpha-synuclein is an endogenous agonist of TLR2 for paracrine activation of microglia. Nat. Commun. 4, 1562 (2013).

16. Ros-Bernal, F. et al. Microglial glucocorticoid receptors play a pivotal role in regulating dopaminergic neurodegeneration in parkinsonism. Proc. Natl Acad. Sci. USA 108, 6632-6637 (2011).

17. Dehmer, T., Heneka, M. T., Sastre, M., Dichgans, J. \& Schulz, J. B. Protection by pioglitazone in the MPTP model of Parkinson's disease correlates with I kappa B alpha induction and block of NF kappa B and iNOS activation. J. Neurochem. 88, 494-501 (2004).

18. Saijo, K. et al. A Nurrl/CoREST pathway in microglia and astrocytes protects dopaminergic neurons from inflammation-induced death. Cell 137, 47-59 (2009).

19. Dai, Y. B., Tan, X. J., Wu, W. F., Warner, M. \& Gustafsson, J. A. Liver X receptor beta protects dopaminergic neurons in a mouse model of Parkinson disease. Proc. Natl Acad. Sci. USA 109, 13112-13117 (2012).

20. Ip, C. W. et al. AAV1/2-induced overexpression of A53T-alpha-synuclein in the substantia nigra results in degeneration of the nigrostriatal system with Lewy-like pathology and motor impairment: a new mouse model for Parkinson's disease. Acta Neuropathol. Commun. 5, 11 (2017).

21. Carrillo-de Sauvage, M. A. et al. Potent and multiple regulatory actions of microglial glucocorticoid receptors during CNS inflammation. Cell Death Differ. 20, 1546-1557 (2013).

22. Tuckermann, J. P. et al. Macrophages and neutrophils are the targets for immune suppression by glucocorticoids in contact allergy. J. Clin. Invest. 117, 1381-1390 (2007).

23. Goldmann, T. et al. A new type of microglia gene targeting shows TAK1 to be pivotal in CNS autoimmune inflammation. Nat. Neurosci. 16, 1618-1626 (2013).

24. Ogawa, S. et al. Molecular determinants of crosstalk between nuclear receptors and Toll-like receptors. Cell 122, 707-721 (2005).

25. Park, B. et al. Proteolytic cleavage in an endolysosomal compartment is required for activation of Toll-like receptor 9. Nat. Immunol. 9, 1407-1414 (2008).

26. Hemmi, H. et al. A Toll-like receptor recognizes bacterial DNA. Nature 408, 740-745 (2000).

27. Hoque, R. et al. A novel small-molecule enantiomeric analogue of traditional (-)-morphinans has specific TLR9 antagonist properties and reduces sterile inflammation-induced organ damage. J. Immunol. 190, 4297-4304 (2013).

28. Shaker, M. E., Trawick, B. N. \& Mehal, W. Z. The novel TLR9 antagonist COV08-0064 protects from ischemia/reperfusion injury in non-steatotic and steatotic mice livers. Biochem. Pharmacol. 112, 90-101 (2016).

29. Deleidi, M., Hallett, P. J., Koprich, J. B., Chung, C. Y. \& Isacson, O. The Tolllike receptor-3 agonist polyinosinic:polycytidylic acid triggers nigrostriatal dopaminergic degeneration. J. Neurosci. 30, 16091-16101 (2010).

30. Turiault, M. et al. Analysis of dopamine transporter gene expression patterngeneration of DAT-iCre transgenic mice. FEBS J. 274, 3568-3577 (2007).

31. Liberatore, G. T. et al. Inducible nitric oxide synthase stimulates dopaminergic neurodegeneration in the MPTP model of Parkinson disease. Nat. Med. 5, 1403-1409 (1999).

32. Boix, J., Padel, T. \& Paul, G. A partial lesion model of Parkinson's disease in mice-characterization of a 6-OHDA-induced medial forebrain bundle lesion. Behav. Brain Res. 284, 196-206 (2015).

33. Shiotsuki, H. et al. A rotarod test for evaluation of motor skill learning. J. Neurosci. Methods 189, 180-185 (2010).

34. Ayton, S. et al. The effect of dopamine on MPTP-induced rotarod disability. Neurosci. Lett. 543, 105-109 (2013).

35. Brinkmann, M. M. et al. The interaction between the ER membrane protein UNC93B and TLR3, 7, and 9 is crucial for TLR signaling. J. Cell Biol. 177, 265-275 (2007).
36. Kim, Y. M., Brinkmann, M. M., Paquet, M. E. \& Ploegh, H. L. UNC93B1 delivers nucleotide-sensing Toll-like receptors to endolysosomes. Nature $\mathbf{4 5 2}$, 234-238 (2008)

37. Ewald, S. E. et al. The ectodomain of Toll-like receptor 9 is cleaved to generate a functional receptor. Nature 456, 658-662 (2008).

38. Sepulveda, F. E. et al. Critical role for asparagine endopeptidase in endocytic Toll-like receptor signaling in dendritic cells. Immunity 31, 737-748 (2009).

39. Park, B. et al. Granulin is a soluble cofactor for Toll-like receptor 9 signaling. Immunity 34, 505-513 (2011).

40. Manoury, B. TLR9 regulation by proteolysis: a friend or a foe. Eur. J. Immunol. 41, 2142-2144 (2011).

41. Sardiello, M. et al. A gene network regulating lysosomal biogenesis and function. Science 325, 473-477 (2009).

42. Zhang, Q. et al. Circulating mitochondrial DAMPs cause inflammatory responses to injury. Nature 464, 104-107 (2010).

43. Oka, T. et al. Mitochondrial DNA that escapes from autophagy causes inflammation and heart failure. Nature 485, 251-255 (2012).

44. Schapira, A. H. \& Jenner, P. Etiology and pathogenesis of Parkinson's disease. Mov. Disord. 26, 1049-1055 (2011)

45. Pan, P. Y. \& Yue, Z. Genetic causes of Parkinson's disease and their links to autophagy regulation. Park. Relat. Disord. 20, S154-S157 (2014).

46. Nadeau, S. \& Rivest, S. Endotoxemia prevents the cerebral inflammatory wave induced by intraparenchymal lipopolysaccharide injection: role of glucocorticoids and CD14. J. Immunol. 169, 3370-3381 (2002).

47. Latz, E. et al. Ligand-induced conformational changes allosterically activate Toll-like receptor 9. Nat. Immunol. 8, 772-779 (2007).

48. Guiducci, C. et al. TLR recognition of self nucleic acids hampers glucocorticoid activity in lupus. Nature 465, 937-941 (2010).

49. Taanman, J. W. The mitochondrial genome: structure, transcription, translation and replication. Biochim. Biophys. Acta 1410, 103-123 (1999).

50. Zhang, W. et al. Aggregated alpha-synuclein activates microglia: a process leading to disease progression in Parkinson's disease. FASEB J. 19, 533-542 (2005).

51. Zecca, L. et al. Human neuromelanin induces neuroinflammation and neurodegeneration in the rat substantia nigra: implications for Parkinson's disease. Acta Neuropathol. 116, 47-55 (2008).

52. Gao, H. M. et al. HMGB1 acts on microglia Mac1 to mediate chronic neuroinflammation that drives progressive neurodegeneration. J. Neurosci. 31 1081-1092 (2011).

53. Santoro, M. et al. In-vivo evidence that high mobility group box 1 exerts deleterious effects in the 1-methyl-4-phenyl-1,2,3,6-tetrahydropyridine model and Parkinson's disease which can be attenuated by glycyrrhizin. Neurobiol. Dis. 91, 59-68 (2016).

54. Podlesniy, P. et al. Mitochondrial DNA in CSF distinguishes LRRK2 from idiopathic Parkinson's disease. Neurobiol. Dis. 94, 10-17 (2016).

55. Kawai, T. \& Akira, S. Toll-like receptors and their crosstalk with other innate receptors in infection and immunity. Immunity 34, 637-650 (2011).

56. Jang, H., Boltz, D. A., Webster, R. G. \& Smeyne, R. J. Viral parkinsonism. Biochim. Biophys. Acta 1792, 714-721 (2009).

57. Rabey, J. M., Scharf, M., Oberman, Z., Zohar, M. \& Graff, E. Cortisol, ACTH, and beta-endorphin after dexamethasone administration in Parkinson's dementia. Biol. Psychiatry 27, 581-591 (1990).

58. Hartmann, A., Veldhuis, J. D., Deuschle, M., Standhardt, H. \& Heuser, I. Twenty-four hour cortisol release profiles in patients with Alzheimer's and Parkinson's disease compared to normal controls: ultradian secretory pulsatility and diurnal variation. Neurobiol. Aging 18, 285-289 (1997).

59. Webster, J. I., Tonelli, L. \& Sternberg, E. M. Neuroendocrine regulation of immunity. Annu. Rev. Immunol. 20, 125-163 (2002).

60. Clausen, B. E., Burkhardt, C., Reith, W., Renkawitz, R. \& Forster, I. Conditional gene targeting in macrophages and granulocytes using LysMcre mice. Transgenic Res. 8, 265-277 (1999).

61. Monier, A., Evrard, P., Gressens, P. \& Verney, C. Distribution and differentiation of microglia in the human encephalon during the first two trimesters of gestation. J. Comp. Neurol. 499, 565-582 (2006).

62. Palva, T. K. \& Palva, E. T. Rapid isolation of animal mitochondrial DNA by alkaline extraction. FEBS Lett. 192, 267-270 (1985).

\section{Acknowledgements}

We are grateful to Drs. S. Parnaudeau and S. O'Regan for helpful comments. We thank IBPS Imaging and Animal platform staff for their technical aid. This work was supported by Association France Parkinson (S.V.), Fondation de France (S.V.), ANR-13-BSV10013-02 (S.V.), ANR TIMMS (F.T.), Fondation pour la Recherche Médicale (Equipe FRM,DEQ 20140329552) and INSERM (ANR 2010 MIDI 00801-B.M.).The "Gene Regualtion and Adaptive Behavior" lab is a member of the LabEX Biological Psychiatry. We thank Prof. Charles C. Duykaerts of NeuroCEB Brain Bank, NeuroCEB Brain Bank has been declared at the French Ministry of Higher Education, and Research and has received approval to distribute post-mortem samples (agreement AC-2013-1887). 


\section{Author contributions}

L.M. performed and analyzed most experiments, as well assisted in writing. A.-C.C. performed and analyzed behavioral experiments, aided in confocal experiments and in human study, M.-A.C.-d.S. and A-P.B. supplied AAV-alpha synuclein vector and assisted in experiments on human tissue, S.L-T. prepared relevant human sections and tissue, V. C., Mi.To., and A.B. assisted in performing experiments, Mi.Tr. and V.B. performed and analyzed the electron microscopy experiments, B.N.T. sent and assisted in TLR9 antagonist experiments, R.M.R. and F.T. gave critical advice and assisted in writing, B.M. gave input in conceiving in vitro experiments, supplied $\mathrm{AEP}^{-1-}$ mice and reagents, as well as assisted in writing, S.V. conceived the study, analyzed the experiments, supervised, and wrote the manuscript.

\section{Additional information}

Supplementary Information accompanies this paper at https://doi.org/10.1038/s41467018-04569-y.

Competing interests: The authors declare no competing interests.

Reprints and permission information is available online at http://npg.nature.com/ reprintsandpermissions/
Publisher's note: Springer Nature remains neutral with regard to jurisdictional claims in published maps and institutional affiliations.

\section{(c) (i)}

Open Access This article is licensed under a Creative Commons Attribution 4.0 International License, which permits use, sharing, adaptation, distribution and reproduction in any medium or format, as long as you give appropriate credit to the original author(s) and the source, provide a link to the Creative Commons license, and indicate if changes were made. The images or other third party material in this article are included in the article's Creative Commons license, unless indicated otherwise in a credit line to the material. If material is not included in the article's Creative Commons license and your intended use is not permitted by statutory regulation or exceeds the permitted use, you will need to obtain permission directly from the copyright holder. To view a copy of this license, visit http://creativecommons.org/ licenses/by/4.0/.

(C) The Author(s) 2018 